\title{
Neuronal Adaptation Effects in Decision Making
}

\author{
Panagiota Theodoni, ${ }^{1}$ Gyula Kovács, ${ }^{2,3}$ Mark W. Greenlee, ${ }^{3}$ and Gustavo Deco ${ }^{1,4}$ \\ ${ }^{1}$ Department of Technology, Computational Neuroscience, Universitat Pompeu Fabra, 08018 Barcelona, Spain, ${ }^{2}$ Department of Cognitive Science, Budapest \\ University of Technology and Economics, H-1111 Budapest, Hungary, ${ }^{3}$ Institute of Psychology, University of Regensburg, 93053 Regensburg, Germany, \\ and ${ }^{4}$ Institució Catalana de Recerca i Estudis Avançats, Universitat Pompeu Fabra, 08010 Barcelona, Spain
}

Recently, there has been an increased interest on the neural mechanisms underlying perceptual decision making. However, the effect of neuronal adaptation in this context has not yet been studied. We begin our study by investigating how adaptation can bias perceptual decisions. We considered behavioral data from an experiment on high-level adaptation-related aftereffects in a perceptual decision task with ambiguous stimuli on humans. To understand the driving force behind the perceptual decision process, a biologically inspired cortical network model was used. Two theoretical scenarios arose for explaining the perceptual switch from the category of the adaptor stimulus to the opposite, nonadapted one. One is noise-driven transition due to the probabilistic spike times of neurons and the other is adaptation-driven transition due to afterhyperpolarization currents. With increasing levels of neural adaptation, the system shifts from a noise-driven to an adaptation-driven modus. The behavioral results show that the underlying model is not just a bistable model, as usual in the decision-making modeling literature, but that neuronal adaptation is high and therefore the working point of the model is in the oscillatory regime. Using the same model parameters, we studied the effect of neural adaptation in a perceptual decision-making task where the same ambiguous stimulus was presented with and without a preceding adaptor stimulus. We find that for different levels of sensory evidence favoring one of the two interpretations of the ambiguous stimulus, higher levels of neural adaptation lead to quicker decisions contributing to a speed-accuracy trade off.

\section{Introduction}

Perceptual decision making is a relevant process in daily life and, during the last few decades, there has been an increased interest in understanding its underlying neural mechanisms (Shadlen and Newsome, 1996; Kim and Shadlen, 1999; Wang, 2002, 2008; Smith and Ratcliff, 2004; Deco and Rolls, 2006; Gold and Shadlen, 2007; Deco et al., 2009). Several theoretical models have been proposed and used, such as diffusion models (Smith and Ratcliff, 2004) and neuronal models (Wang, 2002; Deco and Rolls, 2006; Wong and Wang, 2006, Deco et al., 2009). From these studies, we learn that, in multistable systems, decisions are taken due to noise-driven transitions between attractor states, where noise arises from the random spiking of the neurons.

In the present work, we study a categorical decision process where an ambiguous target stimulus appears with a temporal delay after the presentation of an adaptor stimulus. To this purpose, we consider behavioral data from a high-level, adaptation-related aftereffects experiment (Cziraki et al., 2010). The behavioral data show that prolonged adaptation to

Received May 28, 2010; revised 0ct. 8, 2010; accepted 0ct. 22, 2010.

This work was supported by the European Community's Seventh Framework Programme FP7/2007-2013 under Grant 214728-2 and Bundesministerium für Bildung und Forschung (Project 01GW0653 Brain Plasticity and Perceptual Learning). G.K. was supported by Deutsche Forschungsgemeinschaft Grant K0 3918/1-1. We thank Larissa Albantakis for helpful comments and discussions on the manuscript.

Correspondence should be addressed to Panagiota Theodoni, Department of Technology, Computational Neuroscience, Universitat Pompeu Fabra, Roc Boronat 138, 08018 Barcelona, Spain. E-mail: panagiota. theodoni@upf.edu.

DOI:10.1523/JNEUROSCI.2757-10.2011

Copyright $\odot 2011$ the authors $\quad 0270-6474 / 11 / 310234-13 \$ 15.00 / 0$ a complex stimulus leads to category-specific aftereffects during the presentation of ambiguous stimulus composites. Hypothesizing that neuronal adaptation is relevant for the perceptual judgment, we studied the interaction between noise and neuronal adaptation in this perceptual decisionmaking paradigm. Neuronal adaptation implemented by spike-frequency adaptation reduces the activity of the dominant neuronal population, selective to the adaptor stimulus, leading the nonadapted neuronal population to win the competition when the ambiguous stimulus appears. In contrast, noise-driven fluctuations in the firing rates of the neuronal populations could lead to a switch from one percept to the other. We consider both mechanisms within the same theoretical framework using a spiking neuronal model. We show that neuronal adaptation is the main driving force for the transition from the adaptor category to the novel, opposite category, indicating that the working point of the system is in the oscillatory regime. We thus predict that, if the target ambiguous composite stimulus is presented previously, it will lead to oscillation in perception between the two dissimilar categories. Preliminary experimental evidence confirms our prediction.

Furthermore, we consider, for the first time, the effects of neuronal adaptation in perceptual decision making. To this purpose, we mimic a perceptual task where the same ambiguous composite stimulus appears with and without a preceding adaptor (Cziraki et al., 2010). We simulate this task for different levels of sensory evidence in favor of one of the two dissimilar images and for different levels of neuronal adaptation. We show that, at each level of sensory evidence, neuronal adaptation accelerates the decision process. 


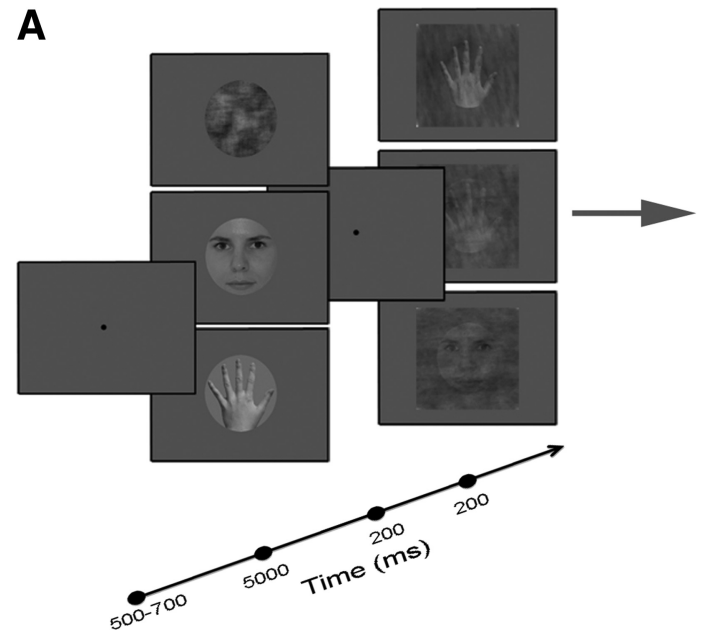

B

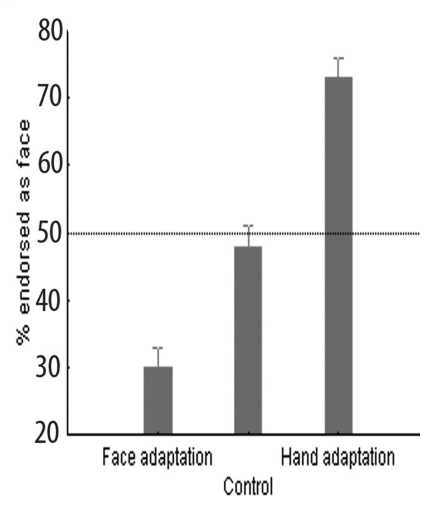

Figure 1. A, Experimental design of the high-level, adaptation-related aftereffects task. Subjects were presented with an adaptor stimulus (face or hand adaptation) for $5 \mathrm{~s}$ and, after a $200 \mathrm{~ms}$ gap (blank screen), with a test stimulus (ambiguous composite image of a face and a hand other than the adaptors) for $200 \mathrm{~ms}$. Subjects reported which image (face or hand) they perceived. $\boldsymbol{B}$, Behavioral adaptation effects. Mean ratio of face responses after face, hand, and control (composite image of face and hand) adaptation for ambiguous face/hand composite test stimuli. Adapted from Cziraki et al. (2010).

Table 1. Tabular description of the spiking model: summary

\begin{tabular}{|c|c|}
\hline Populations & Four: three excitatory, one inhibitory \\
\hline Topology & - \\
\hline Connectivity & Full, no synaptic delay \\
\hline Neuron model & $\begin{array}{l}\text { Leaky integrate-and-fire, fixed voltage threshold, fixed absolute refractory } \\
\text { time }\end{array}$ \\
\hline Channel model & - \\
\hline Synapse model & $\begin{array}{l}\text { Conductance-based AMPA, GABA } \text {, and conductance-based and voltage- } \\
\text { depended NMDA }\end{array}$ \\
\hline Plasticity & - \\
\hline Input & $\begin{array}{l}\text { Time-independent, fixed-rate Poisson spike trains to all neurons: } v=v_{\text {ext }}+ \\
\lambda, v_{\text {ext }}=2.4 \mathrm{kHz} \text {, one generator per neuron }\end{array}$ \\
\hline Measurements & $\begin{array}{l}\text { Spike activity as raster plots, firing rates (calculated in a } 50 \text { ms time window } \\
\text { shifted by } 5 \text { ms step), decision times }\end{array}$ \\
\hline
\end{tabular}

Table 2. Tabular description of the spiking model: population

\begin{tabular}{lll}
\hline Name & Elements & Size \\
\hline $\mathrm{E}_{\text {ext }}$ & Poisson generator & $N_{\mathrm{E}_{\text {ext }}}=800$ \\
$\mathrm{I}$ & IF neuron & $N_{\mathrm{I}}$ \\
$\mathrm{E}=\mathrm{E}_{\mathrm{ns}} \cup \mathrm{H} \cup \mathrm{F}$ & IF neuron & $N_{\mathrm{E}}=4 N_{\mathrm{I}}$ \\
$\mathrm{H}$ & IF neuron & $N_{\mathrm{H}}=f \times N_{\mathrm{E}}$ \\
$\mathrm{F}$ & IF neuron & $N_{\mathrm{F}}=f \times N_{\mathrm{E}}$ \\
$\mathrm{E}_{\text {ns }}$ & IF neuron & $N_{\text {Ens }}=(1-2 f) \times N_{\mathrm{E}}$ \\
\hline
\end{tabular}

\section{Materials and Methods}

Experimental paradigm: high-level adaptation-related aftereffects. In the Cziraki et al. (2010) study of high-level adaptation-related aftereffects, 13 humans ( 8 females) were presented with an adaptor stimulus consisting of a high-contrast female face (face adaptation), a female hand (hand adaptation), or a Fourier randomized version (control condition) for $5 \mathrm{~s}$. After a 200 ms gap (blank screen), an ambiguous, noisy stimulus composite, constructed from an overlapping human face and hand with varying Fourier phase coherences, was shown for $200 \mathrm{~ms}$. Finally, subjects reported which image they perceived (Fig. $1 A$ ). The experimental data indicated that prolonged exposure to complex stimuli, e.g., adaptation to a face or a hand stimulus, biases perceptual decisions toward the nonadapted, dissimilar stimulus category. The simultaneously observed functional magnetic resonance imaging adaptation (fMRIa) effects in the face-sensitive fusiform face area (FFA) and in the body-sensitive extrastriate body area (EBA) were sorted according to the subject's behavioral responses. The fMRIa data indicated that adaptation to the preferred complex stimulus of the given area led to larger signal reduction on trials when it biased category decisions behaviorally than on trials when it was not effective. Figure $1 B$ shows the percentage of trials endorsed as faces for ambiguous face/hand composite test stimuli after face, hand, and control (composite image of face and hand) adaptation. In a control condition, the ambiguous target stimuli were judged $49 \%$ as faces and the remaining times as hands, whereas more trials were judged as faces after hand adaptation (73\%). The opposite is true after face adaptation $(27 \%)$. This shows that prolonged adaptation to a complex stimulus leads to category-specific aftereffects during the perception of ambiguous stimulus composites. fMRI measurements in the areas of interest (FFA and EBA) (Cziraki et al., 2010) supported the behavioral data.

Model description. This paper was written according to the good model description practice proposed by Nordlie et al. (2009). The method that we employed makes use of a biophysically realistic computational model of integrateand-fire neurons that was first introduced by Brunel and Wang (2001) with implemented firing-rate adaptation mechanism (Liu and Wang, 2001). It is based on the attractor paradigm of Amit (1995) and implements competition/cooperation mechanisms among neurons belonging to two neural groups of pyramidal cells that encode the two conflicting interpretations (in a binary decision-making task) or percepts of two dissimilar images of an ambiguous stimulus (in a visual perceptualbistability situation). The essential aspects and components of the model are presented in a compact way in Tables $1-5$ and described below.

In our model, the competing neuronal populations are a handselective neuronal population corresponding to the high-level bodysensitive cortical area EBA (Downing et al., 2001) and a face-selective one corresponding to the fusiform face-sensitive cortical area FFA (Kanwisher et al., 1997). Cooperation mechanisms arise from high recurrent connectivity among neurons belonging to the same neuronal population. Competition between two selective neuronal populations is mediated by a feedback inhibition from interneurons in the network.

Model composition. The network is divided into different populations (Fig. 2A) where neurons belonging to one particular population share the same statistical properties and single-cell parameters, as well as inputs and connectivity. There is a total number of neurons, $N(N=2000$ unless otherwise stated), where $N_{\mathrm{E}}=0.8 \mathrm{~N}$ are excitatory pyramidal cells and $N_{\text {I }}=0.2 N$ are inhibitory interneurons, consistent with the neurophysiologically observed proportion of $80 \%$ pyramidal cells versus $20 \%$ interneurons (Abeles, 1991). The inhibitory neurons form one population to which we will refer as pool I. The excitatory neurons form three distinct populations. Two of them consist of neurons that encode one or the other complex image. Hence, there is a pool $\mathrm{H}$ with neurons, which are selective to the hand images. This neuronal population represents the body-sensitive EBA. There is also a pool $\mathrm{F}$ with neurons selective to the face images. This neuronal population represents the face-sensitive FFA. The remaining excitatory neurons in both areas are nonselective to the particular stimuli ( $p o o l E_{n s}$ ). Nonselective neurons are included for biophysical realism and computationally to increase the stability of the network at the spontaneous state; they do not play a particular role in the decision process per se. The union of these three excitatory populations of neurons is named pool E. Each of the two selective pools ( $\mathrm{H}$ and $\mathrm{F}$ ) consists of $f \times N_{\mathrm{E}}$ neurons. The nonselective one consists of $(1-2 f) \times$ $N_{\mathrm{E}}$ (here $f=0.15$ ). The network is fully connected, meaning that each neuron in the network receives $N_{\mathrm{E}}$ excitatory and $N_{\mathrm{I}}$ inhibitory synaptic contacts. Finally, every neuron receives a background input $\mathrm{E}_{\text {ext }}$. However, we should not necessarily assume that the selective neuronal pools 
Table 3. Tabular description of the spiking model: connectivity

\begin{tabular}{|c|c|c|c|}
\hline Name & Source & Target & Pattern \\
\hline $\mathrm{E}_{\mathrm{ext}}\left(\mathrm{I} / \mathrm{E}_{\mathrm{ns}} / \mathrm{H} / \mathrm{F}\right)$ & $\mathrm{E}_{\mathrm{ext}}$ & $\mathrm{I}, \mathrm{E}_{\mathrm{ns}}, \mathrm{H}, \mathrm{F}$ & $\begin{array}{l}\text { Full connected, weight 1, receptors AMPA, } \\
\text { NMDA }\end{array}$ \\
\hline $\mathrm{I}\left(\mathrm{I} / \mathrm{E}_{\mathrm{ns}} / \mathrm{H} / \mathrm{F}\right)$ & I & $\mathrm{I}, \mathrm{E}_{\mathrm{ns}}, \mathrm{H}, \mathrm{F}$ & Full connected, weight 1, receptors GABA \\
\hline$\left(\mathrm{E}_{\mathrm{ns}} / \mathrm{H} / \mathrm{F}\right) /$ & $\mathrm{E}_{\mathrm{ns}}, \mathrm{H}, \mathrm{F}$ & I & $\begin{array}{l}\text { Full connected, weight 1, receptors AMPA, } \\
\text { NMDA }\end{array}$ \\
\hline$\left(H / F / E_{n s}\right) E_{n s}$ & $H, F, E_{n s}$ & $\mathrm{E}_{\mathrm{ns}}$ & $\begin{array}{l}\text { Full connected, weight 1, receptors AMPA, } \\
\text { NMDA }\end{array}$ \\
\hline $\mathrm{HH}$ & $\mathrm{H}$ & $\mathrm{H}$ & $\begin{array}{l}\text { Full connected, weight } W_{+} \text {, receptors AMPA, } \\
\text { NMDA }\end{array}$ \\
\hline FF & $\mathrm{F}$ & $\mathrm{F}$ & $\begin{array}{l}\text { Full connected, weight } W_{+} \text {, receptors AMPA, } \\
\text { NMDA }\end{array}$ \\
\hline $\mathrm{E}_{\mathrm{ns}}(\mathrm{H} / \mathrm{F})$ & $\mathrm{E}_{\mathrm{ns}}$ & $\mathrm{H}, \mathrm{F}$ & $\begin{array}{l}\text { Full connected, weight } W_{-} \text {, receptors AMPA, } \\
\text { NMDA }\end{array}$ \\
\hline HF & $\mathrm{H}$ & $\mathrm{F}$ & $\begin{array}{l}\text { Full connected, weight } W_{-} \text {, receptors AMPA, } \\
\text { NMDA }\end{array}$ \\
\hline $\mathrm{FH}$ & $\mathrm{F}$ & $\mathrm{H}$ & $\begin{array}{l}\text { Full connected, weight } W_{-} \text {, receptors AMPA, } \\
\text { NMDA }\end{array}$ \\
\hline
\end{tabular}

Table 4. Tabular description of the spiking model: neuron and synapse models

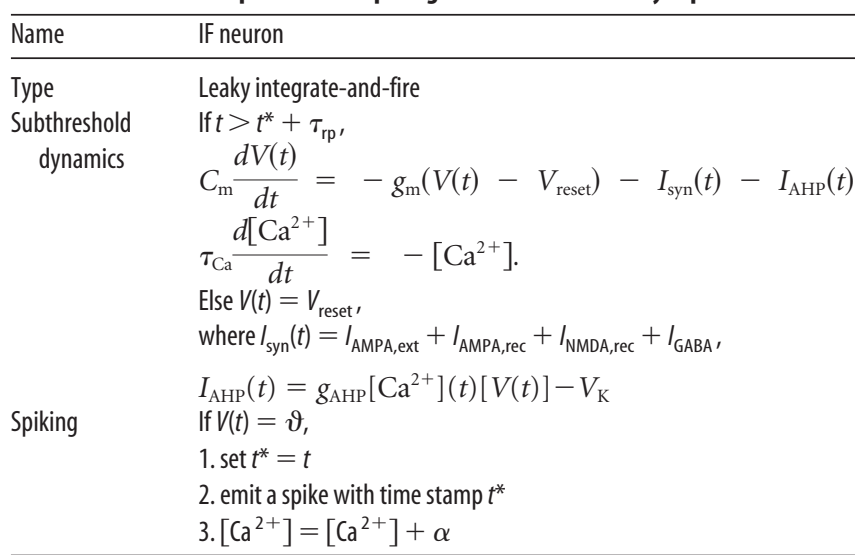

correspond to the cortical areas FFA and EBA. In fact, the process studied with our model could happen in another brain region and then project the result to the FFA and EBA so that top-down interaction effects could also account for Cziraki et al.'s (2010) findings.

Connectivity. The connectivity architecture of the network can be seen graphically in Figure $2 \mathrm{~A}$. The connections of the neurons and their strengths between and within the populations are determined by dimensionless weights $W_{j}$, corresponding to the synaptic efficacies relatively to a baseline, which is set to 1 . The recurrent self-excitation within the selective neuronal populations $\mathrm{H}$ and $\mathrm{F}$ is given by the weight $W_{+}$and the weaker connection between them by the weight $W_{-}$. The remaining connections are all set to the value of the baseline, i.e., to 1 . These values are fixed and assumed to have developed by a Hebbian learning mechanism, according to which they are high when the activity of presynaptic and postsynaptic neurons is correlated (e.g., $W_{+}>1$ ), low when it is anticorrelated $\left(W_{-}<1\right)$, and equal to the baseline when it is uncorrelated. The synaptic efficacy $W_{-}$depends on $W_{+}$by the relation $W_{-}=$ $1-f\left(W_{+}-1\right) /(1-f)$ to ensure that, during the simulation process, the average excitatory synaptic efficacy remains constant.

Dynamics of neurons, synapses and channels. All neurons in the network are modeled as being leaky integrate-and-fire neurons. Integrate-andfire (IF) neurons are point-like elements, meaning that the whole neural membrane is taken as equipotential. Its dynamical state is described by a single variable, the instantaneous value of its membrane potential, $V(t)$. Under normal conditions, the potential inside the cell is lower than the potential outside, which is zero by convention. Membrane voltage changes are due to different ionic concentrations found on both sides of the membrane. Synaptic inputs to an IF neuron are basically described by a capacitor, $C_{\mathrm{m}}$, connected in parallel with a resistor, $R_{\mathrm{m}}$. The capacitor corresponds to the membrane capacitance and the resistor to the membrane conductance through which currents are injected into the neuron by its synapses $\left(I_{\text {syn }}\right)$. In addition, in this work a spike-frequency adapting mechanism is taken into account, which has been observed experimentally (Madison and Nicoll, 1984). It is implemented in the network by including a slow $\mathrm{Ca}^{2+}$-activated $\mathrm{K}^{+}$current $I_{\mathrm{AHP}}$ into the dynamical equation of the membrane potential of each neuron. This afterhyperpolarization current $I_{\mathrm{AHP}}$ is given by the following equation:

$$
\left.I_{\mathrm{AHP}}(t)=-g_{\mathrm{AHP}}\left[\mathrm{Ca}^{2+}\right](t)\left[V(t)-V_{\mathrm{K}}\right)\right],
$$

where $V_{\mathrm{K}}=-80 \mathrm{mV}$ is the reversal potential of potassium channels. The cytoplasmic $\mathrm{Ca}^{2+}$ concentration, $\left[\mathrm{Ca}^{2+}\right]$, is initially set to $0 \mu \mathrm{M}$ and between spikes is modeled as a leaky integrator with a decay time constant $\tau_{\mathrm{Ca}}=600 \mathrm{~ms}$ (Liu and Wang, 2001). The $g_{\mathrm{AHP}}\left[\mathrm{Ca}^{2+}\right]$ is the effective $\mathrm{K}^{+}$conductance.

Hence, the dynamics of the subthreshold membrane potential of each neuron in the network is given by the following system of equations:

$$
\begin{gathered}
C_{\mathrm{m}} \frac{d V(t)}{d t}=-g_{\mathrm{m}}\left(V(t)-V_{\mathrm{L}}\right)-I_{\text {syn }}(t)+I_{\mathrm{AHP}}(t) \\
\tau_{\mathrm{Ca}} \frac{d\left[\mathrm{Ca}^{2+}\right]}{d t}=-\left[\mathrm{Ca}^{2+}\right],
\end{gathered}
$$

where $C_{\mathrm{m}}=0.5 \mathrm{nF}$ for excitatory neurons and $C_{\mathrm{m}}=0.2 \mathrm{nF}$ for inhibitory neurons, $g_{\mathrm{m}}=1 / R_{\mathrm{m}}$ is the membrane leak conductance with the values $g_{\mathrm{m}}=25 \mathrm{nS}$ for pyramidal cells and $g_{\mathrm{m}}=20 \mathrm{nS}$ for interneurons, $V_{\mathrm{L}}=$ $-70 \mathrm{mV}$ is the resting potential, and $I_{\text {syn }}$ is the synaptic current entering the neuron.

When the membrane potential reaches a certain threshold, $V_{\mathrm{thr}}=\vartheta=$ $-50 \mathrm{mV}$, a spike is emitted and transmitted to other neurons. Next, the membrane potential is reset to $V_{\text {reset }}=-55 \mathrm{mV}$ after a refractory time, $\tau_{\text {ref }}=2 \mathrm{~ms}$ for excitatory neurons and $\tau_{\text {ref }}=1 \mathrm{~ms}$ for inhibitory ones, during which the neuron is unable to produce further spikes. In addition, the cytoplasmic $\mathrm{Ca}^{2+}$ concentration increases by a small amount, $\alpha=$ $0.005 \mu \mathrm{M}$. Therefore, if $V(t)=V_{\mathrm{thr}}$, a spike is emitted and

$$
\left\{\begin{array}{l}
V \rightarrow V_{\text {reset }} \\
{\left[\mathrm{Ca}^{2+}\right] \rightarrow\left[\mathrm{Ca}^{2+}\right]+\alpha .}
\end{array}\right.
$$

The values of the constant parameters and default values of the free parameters used in the simulations are displayed in Table 5. They are equal to the ones used in the network introduced by Brunel and Wang (2001).

Ion channels are modeled as having three types of receptors mediating the synaptic currents flowing into them: AMPA and NMDA glutamatergic and $\mathrm{GABA}_{\mathrm{A}}$ GABAergic receptors. The total synaptic current of a neuron is given by the sum of the recurrent EPSCs, mediated by slow NMDA and fast AMPA receptors ( $I_{\mathrm{NMDA}, \mathrm{rec}}$ and $I_{\mathrm{AMPA}, \mathrm{rec}}$, respectively), and the IPSCs, mediated by $\mathrm{GABA}_{\mathrm{A}}$ receptors $\left(I_{\mathrm{GABA}}\right)$. External cells contribute to the current through AMPA receptors $\left(I_{\mathrm{AMPA}, \mathrm{ext}}\right)$. External EPSCs were mediated exclusively by AMPA receptors to keep the external stimulus as simple as possible. Recurrent EPSCs include NMDA receptors because it is helpful to maintain the system in an asynchronous state. Hence, the total synaptic current is given by $I_{\text {syn }}(t)=I_{\mathrm{AMPA}, \text { ext }}+$ $I_{\mathrm{AMPA}, \mathrm{rec}}+I_{\mathrm{GABA}}$, where:

$$
\begin{gathered}
I_{\mathrm{AMPA}, \mathrm{ext}}(t)=g_{\mathrm{AMPA}, \mathrm{ext}}\left(V(t)-V_{\mathrm{E}}\right) \sum_{j=1}^{N_{\text {ext }}} s_{j}^{\mathrm{AMPA}, \mathrm{ext}}(t) \\
I_{\mathrm{AMPA}, \mathrm{rec}}(t)=g_{\mathrm{AMPA}, \mathrm{rec}}\left(V(t)-V_{\mathrm{E}}\right) \sum_{j=1}^{N_{\mathrm{E}}} W_{j} s_{j}^{\mathrm{AMPA}, \mathrm{rec}}(t) \\
I_{\mathrm{NMDA}, \mathrm{rec}}(t)=\frac{g_{\mathrm{NMDA}, \mathrm{rec}}\left(V(t)-V_{\mathrm{E}}\right)}{1+\gamma e^{-\beta V(t)}} \sum_{j=1}^{N_{\mathrm{E}}} W_{j} s_{j}^{\mathrm{NMDA}, \mathrm{rec}}(t)
\end{gathered}
$$


Table 5. Default values of the parameters used in the simulations

\begin{tabular}{|c|c|c|c|c|c|}
\hline \multicolumn{2}{|c|}{ Excitatory and inhibitory neurons } & \multicolumn{2}{|c|}{ Excitatory neurons } & \multicolumn{2}{|c|}{ Inhibitory neurons } \\
\hline$N$ & 2000 neurons & $N_{\text {Ens }}$ & 1120 neurons & $N_{\mathrm{I}}$ & 400 neurons \\
\hline$N_{\text {ext }}$ & 800 neurons & $V_{E}$ & $0 \mathrm{mV}$ & $V_{1}$ & $-70 \mathrm{mV}$ \\
\hline$f$ & 0.15 & $C_{m}$ & $0.5 \mathrm{nF}$ & $C_{m}$ & $0.2 \mathrm{nF}$ \\
\hline$v_{\text {ext }}$ & $2.4 \mathrm{kHz}$ & $g_{\mathrm{m}}$ & $25 \mathrm{nS}$ & $g_{\mathrm{m}}$ & $20 \mathrm{nS}$ \\
\hline$V_{\mathrm{L}}$ & $-70 \mathrm{mV}$ & $\tau_{\text {ref }}$ & $2 \mathrm{~ms}$ & $\tau_{\text {ref }}$ & $1 \mathrm{~ms}$ \\
\hline$V_{\text {thr }}$ & $-50 \mathrm{mV}$ & $g_{\mathrm{AMPA}, \mathrm{ext}}$ & $2.08 \mathrm{nS}$ & $g_{\mathrm{AMPA}, \mathrm{ext}}$ & $1.62 \mathrm{nS}$ \\
\hline$V_{\text {reset }}$ & $-55 \mathrm{mV}$ & $g_{\mathrm{AMPA}, \mathrm{rec}}$ & $104 / N=0.052 \mathrm{nS}$ & $g_{\mathrm{AMPA}, \mathrm{rec}}$ & $81 / N=0.0405 \mathrm{nS}$ \\
\hline$W_{+}$ & 1.65 & $g_{\mathrm{NMDA}}$ & $327 / N=0.1635 \mathrm{nS}$ & $g_{\mathrm{NMDA}}$ & $258 / N=0.129 \mathrm{nS}$ \\
\hline$g_{\text {AHP }}$ & $10 \mathrm{nS}$ & $g_{\mathrm{GABA}}$ & $0.625 \mathrm{nS}$ & $g_{\mathrm{GABA}}$ & $0.4865 \mathrm{nS}$ \\
\hline$\alpha$ & $0.005 \mu \mathrm{M}$ & $N_{\mathrm{H}}=N_{\mathrm{F}}$ & 240 neurons & & \\
\hline$\tau_{\mathrm{CA}}$ & $600 \mathrm{~ms}$ & $\lambda_{\text {adaptor }}$ & $200 \mathrm{~Hz}$ & & \\
\hline$t_{\mathrm{AMPA}}$ & $2 \mathrm{~ms}$ & $\lambda_{\mathrm{H}}=\lambda_{\mathrm{F}}=\lambda$ & $50 \mathrm{~Hz}$ & & \\
\hline$t_{\mathrm{NMDA} \uparrow}$ & $2 \mathrm{~ms}$ & & & & \\
\hline$t_{\mathrm{NMDA} \downarrow} \downarrow$ & $100 \mathrm{~ms}$ & & & & \\
\hline$t_{\mathrm{GABA}}$ & $10 \mathrm{~ms}$ & & & & \\
\hline
\end{tabular}

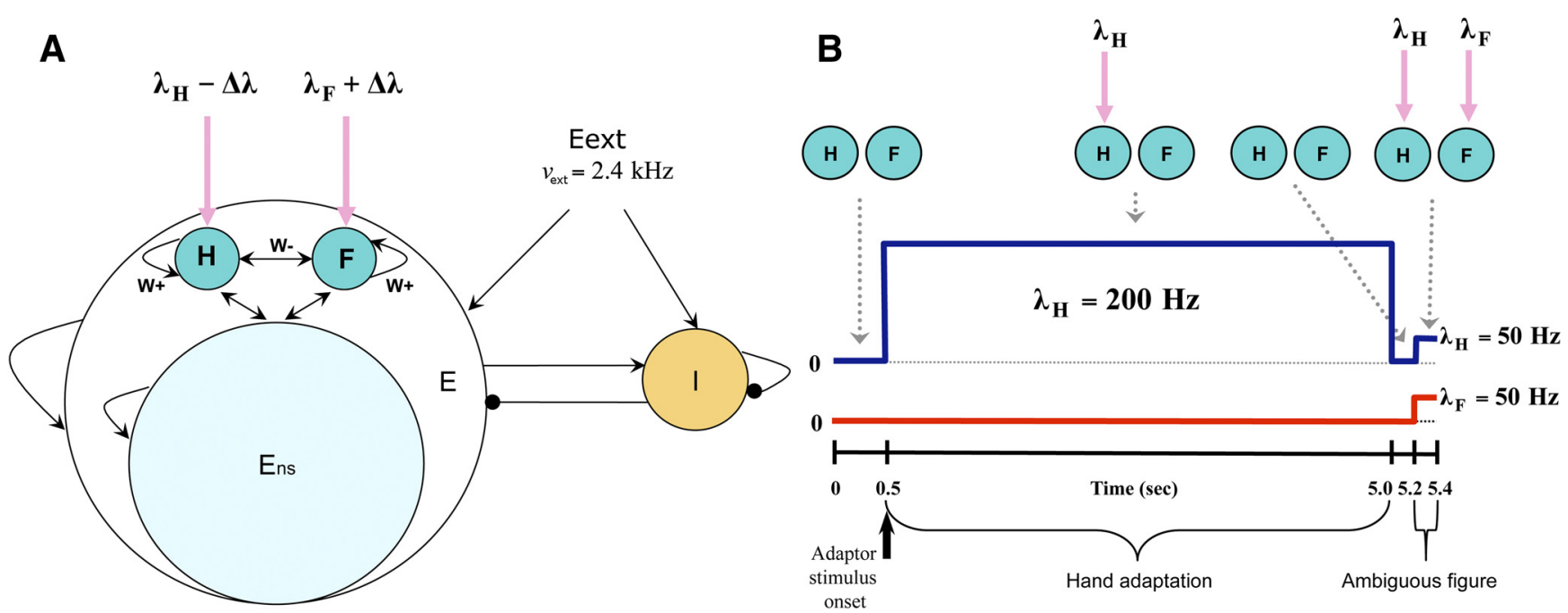

Figure 2. Architecture of the network and stimulation protocol mimicking Cziraki et al.'s (2010) experiment. $\boldsymbol{A}$, Architecture of the network. The size of neuronal populations is proportional to their number of neurons $\left(N_{\mathrm{E}}=2 N_{\text {ext }}=4 N_{\mathrm{I}}=N_{\mathrm{Ens}}+N_{\mathrm{H}}+N_{\mathrm{F}} N_{\mathrm{Ens}}=0.7 N_{\mathrm{E}}\right.$ and $\left.N_{\mathrm{H}}=N_{\mathrm{F}}=0.15 N_{\mathrm{E}}\right)$. B, Stimulation protocol. During the first 500 ms, the external input to both pools is $\lambda_{\mathrm{H}}=$ $\lambda_{\mathrm{F}}=0 \mathrm{~Hz}$ to represent the absence of competing stimuli in the first stage of the psychophysical task. During this period, the network exhibits spontaneous activity ( $3 \mathrm{~Hz}$ for the excitatory pools and $9 \mathrm{~Hz}$ for the inhibitory pools). For the next $4500 \mathrm{~ms}$, we set the value of the external stimulus to the pool corresponding to the hand stimulus equal to $\lambda_{\mathrm{H}}=200 \mathrm{~Hz}$ and $\lambda_{\mathrm{F}}=0 \mathrm{~Hz}$, corresponding to the hand adaptation process. For a period of $200 \mathrm{~ms}$, both external stimuli are set to zero, $\lambda_{\mathrm{H}}=\lambda_{\mathrm{F}}=0 \mathrm{~Hz}$, which corresponds to the postadaptation blank period used by Cziraki et al. (2010). For the last $200 \mathrm{~ms}$, both are set to $50 \mathrm{~Hz}, \lambda_{\mathrm{H}}=\lambda_{\mathrm{F}}=50 \mathrm{~Hz}$, which corresponds to the presentation of the ambiguous test stimulus (composite image of a hand and a face). The colored circles represent the different neural populations.

$$
I_{\mathrm{GABA}}(t)=g_{\mathrm{GABA}}\left(V(t)-V_{\mathrm{I}}\right) \sum_{j=1}^{N_{\mathrm{I}}} s_{j}^{\mathrm{GABA}}(t) .
$$

The reversal potentials for EPSCs are $V_{\mathrm{E}}=0 \mathrm{mV}$ and for IPSCs, $V_{\mathrm{I}}=-70$ $\mathrm{mV}$. The dimensionless parameters $W_{j}$ of the excitatory connections are the synaptic weights. The synaptic conductances for excitatory neurons are $g_{\mathrm{AMPA}, \mathrm{ext}}=2.08 \mathrm{nS}, g_{\mathrm{AMPA}, \mathrm{rec}}=104 / \mathrm{N} \mathrm{nS}, g_{\mathrm{NMDA}, \mathrm{rec}}=327 / \mathrm{N} \mathrm{nS}$, and $g_{\mathrm{GABA}}=1250 / \mathrm{N} \mathrm{nS}$, where $\mathrm{N}$ is the total number of neurons in the network and divides the recurrent conductances to keep the mean recurrent input constant if $\mathrm{N}$ is varied in the network. The synaptic conductances for inhibitory neurons are $g_{\mathrm{AMPA}, \text { ext }}=1.62 \mathrm{nS}, g_{\mathrm{AMPA}, \mathrm{rec}}=81 / \mathrm{N}$ $\mathrm{nS}, g_{\mathrm{NMDA}, \mathrm{rec}}=258 / \mathrm{N} \mathrm{nS}$, and $g_{\mathrm{GABA}}=973 / \mathrm{N} \mathrm{nS}$. The NMDA currents are voltage-dependent and they are modulated by intracellular magnesium concentration $\left[\mathrm{Mg}^{2+}\right]=1.0 \mathrm{mM}$, with parameters $\gamma=\left[\mathrm{Mg}^{2+}\right] /$ (3.57 mM) and $\beta=0.062(\mathrm{mV})^{-1}$. The functions $s_{j}^{i}(t)$ represent the fractions of open channels of neurons and they are given by the following:

$$
\frac{d s_{j}^{\mathrm{AMPA}, \mathrm{ext}}(t)}{d t}=-\frac{s_{j}^{\mathrm{AMPA}, \mathrm{ext}}(t)}{\tau_{\mathrm{AMPA}}}+\sum_{k} \delta\left(t-t_{j}^{k}\right)
$$

$$
\begin{gathered}
\frac{d s_{j}^{\mathrm{AMPA}, \mathrm{rec}}(t)}{d t}=-\frac{s_{j}^{\mathrm{AMPA}, \mathrm{rec}}(t)}{\tau_{\mathrm{AMPA}}}+\sum_{k} \delta\left(t-t_{j}^{k}\right) \\
\frac{d s_{j}^{\mathrm{NMDA}, \mathrm{rec}}(t)}{d t}=-\frac{s_{j}^{\mathrm{NMDA}, \mathrm{rec}}(t)}{\tau_{\mathrm{NMDA} \downarrow}}+\alpha x_{j}(t)\left(1-s_{j}^{\mathrm{NMDA}, \mathrm{rec}}(t)\right) \\
\frac{d x_{j}(t)}{d t}=-\frac{x_{j}(t)}{\tau_{\mathrm{NMDA} \uparrow}}+\sum_{k} \delta\left(t-t_{j}^{k}\right) \\
\frac{d s_{j}^{\mathrm{GABA}}(t)}{d t}=-\frac{s_{j}^{\mathrm{GABA}}(t)}{\tau_{\mathrm{GABA}}}+\sum_{k} \delta\left(t-t_{j}^{k}\right),
\end{gathered}
$$

where sums over $k$ represent sums over spikes emitted by the presynaptic neuron $j$ at time $t_{j}^{k} ; \alpha=0.5 \mathrm{~ms}^{-1}$ and $\delta(t)$ is the Dirac delta function. The rise time for the NMDA synapses is $t_{\mathrm{NMDA} \uparrow}=2 \mathrm{~ms}$. The rise times of both AMPA and GABA synaptic currents are neglected because they are extremely short $(<1 \mathrm{~ms})$. The decay time for AMPA synapses is $t_{\mathrm{AMPA}}=$ 
$2 \mathrm{~ms}$, for NMDA synapses is $t_{\mathrm{NMDA} \downarrow}=100 \mathrm{~ms}$, and for GABA synapses is $t_{\mathrm{GABA}}=10 \mathrm{~ms}$.

Model input and output. The external background input is given by uncorrelated Poisson spike trains from 800 external excitatory connections from neurons firing at a rate of $3 \mathrm{~Hz}$. Hence, the excitatory and the inhibitory neurons of the network receive an independent Poisson train of spikes with rate $v_{\mathrm{ext}}=2.4 \mathrm{kHz}$. In addition, to represent the sensory stimulus, meaning presentation of the hand and/or face stimuli, the neurons belonging to the two selective populations ( $\mathrm{H}$ and $\mathrm{F}$ ) receive an additional Poisson spike train with invariant time rates $\lambda_{\mathrm{H}}$ and $\lambda_{\mathrm{F}}$, respectively. Input $\lambda_{\mathrm{H}}$ corresponds to activation of the neurons of the cortical area EBA due to presentation of the hand image and $\lambda_{\mathrm{F}}$ corresponds to activation of the neurons of the cortical area FFA due to presentation of the face image. Therefore, the total input that each neuron of the selective pools receives is $v_{\mathrm{H}, \mathrm{F}}=v_{\mathrm{ext}}+\lambda_{\mathrm{H}, \mathrm{F}}$. To simulate the presentation of the ambiguous composite image of a hand and a face, the same external input is applied to the two selective neuronal populations: $\lambda_{\mathrm{H}}=$ $\lambda_{\mathrm{F}} \equiv \lambda \Leftrightarrow v_{\mathrm{H}}=v_{\mathrm{F}}$. Throughout this work, $\lambda=50 \mathrm{~Hz}$ unless otherwise stated. To simulate the adaptor stimulus, we add an external input to one of the selective pools, e.g., for $\mathrm{H}$ pool: $\lambda_{\mathrm{H}}=\lambda_{\text {adaptor }}$, whereas the competitive one receives only background input $v_{\text {ext }}$. Throughout this study, $\lambda_{\text {adaptor }}=200 \mathrm{~Hz}$ unless otherwise stated. We followed various stimulation protocols, which are described in detail below. Output of the model is the mean spike activity of the neuronal populations of the network, as are raster plots and firing rates. From the firing rates of the two selective pools, we also calculate the decision times. In the simulations, the total number of neurons of the network is 2000 unless otherwise stated.

Free parameters of the model. All free parameters of the network are presented in Table 5 with their default values. If they are different, it is declared where necessary. We stress that the parameters with which we manipulate in this work, i.e., the free parameters, are the total number of neurons $(N)$, the external inputs $\left[\left(\lambda_{\mathrm{H}}, \lambda_{\mathrm{F}}\right) \Rightarrow \lambda_{\text {adaptor }}, \lambda\right]$, the level of the adaptation that is regulated by $\mathrm{K}^{+}$conductance, $\mathrm{g}_{\mathrm{AHP}}$, and the recurrent synaptic connectivity $W_{+}$of the selective neuronal populations $\mathrm{H}$ and $\mathrm{F}$.

Stimulation protocol A: bifurcation diagrams. The spiking model used in this work exhibits distinct regimes depending on the free parameters. To gain intuition about how the system transits from one regime to the other, we calculated the firing rate of one selective population first as a function of neuronal adaptation and later as a function of external stimulus. For this purpose, we simulated a task where an ambiguous stimulus [like Cziraki et al.'s (2010) ambiguous composite image of a hand and a face] is presented continuously in time. Here the number of total neurons of the network is 4000 to eliminate the noise of the system. In this model, noise cannot be explicitly excluded since it arises from the finite number of neurons in the network. Therefore, we performed the same simulation, increasing the total number neurons with a 500 neurons step and in the range of $500 \leq N \leq 4500$, each time defining the bifurcation point, $g_{\text {bif }}$. We saw that for $N \geq 3000$, the value of $g_{\text {bif }}$ did not change $>1 \%$. The neurons of selective pools $\mathrm{H}$ and $\mathrm{F}$, for this task, receive a Poisson spike train with an invariant firing rate of: $v_{\mathrm{H}}=v_{\mathrm{F}}=v_{\mathrm{ext}}+0 \mathrm{~Hz}$ for $0 \leq t<500 \mathrm{~ms}$ and $v_{\mathrm{H}}=v_{\mathrm{F}}=v_{\mathrm{ext}}+\lambda \mathrm{Hz}$ for $500 \leq t<10,000 \mathrm{~ms}$.

We performed this simulation for different levels of adaptation corresponding to the conductance $g_{\mathrm{AHP}}$, ranging from 0 to $g_{\mathrm{AHP}, \max }=12 \mathrm{nS}$ for $W_{+}=1.65$ and $\lambda=50 \mathrm{~Hz}$, to show how the system transits from bistability to an oscillatory regime. In addition, we simulated the same task for different levels of external stimulus $\lambda$ (neuronal adaptation $g_{\mathrm{AHP}}=10 \mathrm{nS}$ and recurrent connectivity $\left.W_{+}=1.65\right)$ to show how the system transits from a spontaneous state to an oscillatory regime. Spontaneous state is defined as the state when the firing rates of both selective pools are $<10 \mathrm{~Hz}$. The parameter space of $W_{+}$and $g_{\mathrm{AHP}}$ for which the system remains in the spontaneous state in the absence of external input (Fig. 3 ) is described in detail in Parameter range of $W_{+}$and $g_{\mathrm{AHP}}$, below. This parameter space also describes the dependence of the upper limit of the level of neuronal adaptation, $g_{\mathrm{AHP} \text {,max }}$, on the recurrent synaptic weight, $W_{+}$, of the selective populations.

Stimulation protocol B: replication of the Cziraki et al. (2010) experiment. To replicate the experimental design under discussion, during the first $500 \mathrm{~ms}$ the external input to both pools is $\lambda_{\mathrm{H}}=\lambda_{\mathrm{F}}=0 \mathrm{~Hz}$, representing the absence of competing stimuli in the first stage of the experi-

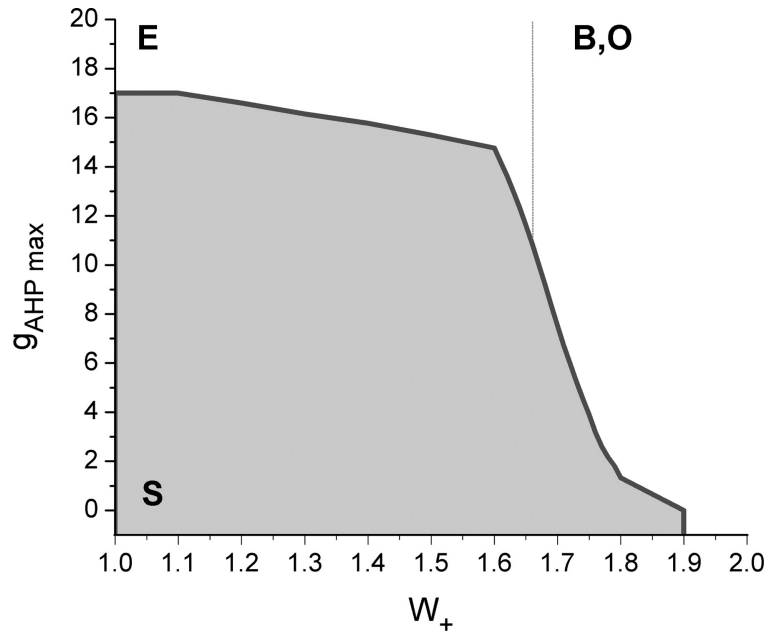

Figure 3. Parameter space of recurrent connectivity $W_{+}$weights and afterhyperpolarization conductance $g_{\text {AHP }}$ when no additional external input is given to the selective neuronal populations of the network, except the background input, with the constraint that the network should stay in the spontaneous state. The parameter space is separated into two regions by the thick line. The shaded region shows where the network remains in the spontaneous state (S) and the unshaded area shows where it does not. The thin line divides the parameter space in two smaller regions. The excitation region $(\mathrm{E})$, where both pools are excited and no decisions can be taken, and the bistability (B)/oscillatory (0) region where recurrent connectivity is big enough that low levels of adaptation excite the network to decisions or oscillatory states, even without additional external input. These regions are not acceptable and thus these values were not taken into account in the parameter search.

mental task. During this period, the network exhibits spontaneous activity of $3 \mathrm{~Hz}$ for excitatory pools and $9 \mathrm{~Hz}$ for the inhibitory pool. For the next $4500 \mathrm{~ms}$, which is the period of presentation of adaptor stimulus, we set the value of external stimulus to the pool corresponding to the hand image equal to $\lambda_{\mathrm{H}}=\lambda_{\text {adaptor }}=200 \mathrm{~Hz}$ and set the external input to pool $\mathrm{F}$ as $\lambda_{\mathrm{F}}=0 \mathrm{~Hz}$. Then both external inputs are set to $0 \mathrm{~Hz}\left(\lambda_{\mathrm{H}}=\lambda_{\mathrm{F}}=\right.$ $0 \mathrm{~Hz}$ ) for a period of $200 \mathrm{~ms}$ and to $50 \mathrm{~Hz}$ for the last $200 \mathrm{~ms}$ of the cue $\left(\lambda_{\mathrm{H}}=\lambda_{\mathrm{F}} \equiv \lambda=50 \mathrm{~Hz}\right)$, which corresponds to the presentation of the ambiguous composite image of a hand and a face. Summarizing the stimulation protocol in this case (Fig. $2 B$ ) is as follows:

$$
\begin{gathered}
\nu_{\mathrm{H}}=\nu_{\mathrm{F}}=\nu_{\text {ext }}+0 \mathrm{~Hz} \quad \text { for } 0 \leq t<500 \mathrm{~ms} \\
\left.\begin{array}{c}
\nu_{\mathrm{H}}=\nu_{\text {ext }}+\lambda_{\text {adaptor }} \\
\nu_{\mathrm{F}}=\nu_{\text {ext }}+0 \mathrm{~Hz}
\end{array}\right\} \quad \text { for } 500 \leq t<5000 \mathrm{~ms} \\
\nu_{\mathrm{H}}=\nu_{\mathrm{F}}=\nu_{\text {ext }}+0 \mathrm{~Hz} \text { for } 5000 \leq t<5200 \mathrm{~ms} \\
\nu_{\mathrm{H}}=\nu_{\mathrm{F}}=\nu_{\text {ext }}+\lambda \quad \text { for } 5200 \leq t \leq 5400 \mathrm{~ms} .
\end{gathered}
$$

The total number of neurons of the network is 2000 to take into account the noise of the network. The recurrent synaptic connectivity is $W_{+}=$ 1.65. We performed 100 trials and calculated the mean ratio of face and hand responses after hand adaptation in the EBA for each value of the level of adaptation, $g_{\mathrm{AHP}}$. We did the same for 10 series of 100 trials and calculated the mean percentage performance at each. We averaged over the 10 series and calculated the SDs. A face response occurs when the firing rate of selective pool $\mathrm{F}$ at $t=5400 \mathrm{~ms}$ is $>10 \mathrm{~Hz}$ (because spontaneous state is typically below this value) and surpasses the firing rate of selective pool $\mathrm{H}$ at the same time by at least $5 \mathrm{~Hz}$. Hand responses were defined as non-face responses, i.e., total number of trials minus number of face responses.

Stimulation protocol C: decision making, reaction time. In this part of our study, we examined the effects of neuronal adaptation in a binary decision-making task. To this purpose, we simulated an experiment similar to Cziraki et al. (2010) with and without a preceding adaptor. The proposed experiment corresponds to the presentation of an ambiguous composite image of a hand and face that is gradually changed over trials to one of the two interpretations by increasing the contrast of one image 
(e.g., the face) in different blocks of trials. We simulated this by introducing a bias between the external inputs to selective pools $\mathrm{H}$ and $\mathrm{F}$ by increasing the level of sensory evidence to pool $\mathrm{F}$ and decreasing it to pool $\mathrm{H}$ at each set of simulations. Decision-making, without adaptor, is shown as:

$$
\begin{gathered}
\nu_{\mathrm{H}}=\nu_{\mathrm{F}}=\nu_{\mathrm{ext}} \quad \text { for } 0 \leq t \leq 3000 \mathrm{~ms} \\
\left.\begin{array}{c}
\nu_{\mathrm{H}}=\nu_{\text {ext }}+\lambda-\Delta \lambda \\
\nu_{\mathrm{F}}=\nu_{\text {ext }}+\lambda+\Delta \lambda
\end{array}\right\} \quad \text { for } 3000 \leq t \leq 6500 \mathrm{~ms} .
\end{gathered}
$$

We refer to this stimulation protocol as decision making without adaptor because there is no stimulation of one selective pool preceding the decision-making task. We also examined the model with an adaptor stimulus preceding the target [i.e., where pool $\mathrm{H}$ is stimulated initially as in Cziraki et al.'s (2010) experiment]. Decision-making, with adaptor, is shown as:

$$
\begin{gathered}
\nu_{\mathrm{H}}=\nu_{\mathrm{F}}=\nu_{\mathrm{ext}} \quad \text { for } 0 \leq t<500 \mathrm{~ms} \\
\left.\begin{array}{c}
\nu_{\mathrm{H}}=\nu_{\mathrm{ext}}+\lambda_{\text {adaptor }} \\
\nu_{\mathrm{F}}=\nu_{\mathrm{ext}}
\end{array}\right\} \quad \text { for } 500 \leq t<5000 \mathrm{~ms} \\
\nu_{\mathrm{H}}=\nu_{\mathrm{F}}=\nu_{\mathrm{ext}} \quad \text { for } 5000 \leq t<5200 \mathrm{~ms} \\
\left.\begin{array}{c}
\nu_{\mathrm{H}}=\nu_{\mathrm{ext}}+\lambda-\Delta \lambda \\
\nu_{\mathrm{F}}=\nu_{\mathrm{ext}}+\lambda+\Delta \lambda
\end{array}\right\} \quad \text { for } 5200 \leq t<6500 \mathrm{~ms} .
\end{gathered}
$$

We performed 1000 simulations for the decision-making task without adaptor, and 100 simulations for the decision-making task with adaptor. In both cases, we calculated the mean firing rates of both selective populations, the mean time needed for pool $\mathrm{F}$ to win the competition with pool $\mathrm{H}$, and the percentage of correct performance on correct trials (when pool $\mathrm{F}$ won pool $\mathrm{H}$ ) as a function of $\Delta \mathrm{I}$, where $\Delta I=2 \times \Delta \lambda$. In addition, we performed the same simulations varying the level of adaptation. In the decision-making task without a preceding adaptor, neuronal adaptation is varied within the range $0 \leq g_{\mathrm{AHP}} \leq 12 \mathrm{nS}$, whereas $W_{+}=1.65$ and $\lambda=50 \mathrm{~Hz}$. In the decision-making task with a preceding adaptor, we studied three different levels of neuronal adaptation, where for each level the remaining free parameters were the same as those used in the model coinciding with the Cziraki et al. (2010) experiment $\left(W_{+}=\right.$ $1.65, \lambda=50 \mathrm{~Hz}, \lambda_{\text {adaptor }}=300 \mathrm{~Hz}$ for $g_{\mathrm{AHP}}=9 \mathrm{nS}, \lambda_{\text {adaptor }}=200 \mathrm{~Hz}$ for $g_{\mathrm{AHP}}=10 \mathrm{nS}$, and $\lambda_{\text {adaptor }}=160 \mathrm{~Hz}$ for $g_{\mathrm{AHP}}=11 \mathrm{nS}$ ). When the system is in the bistable region, a correct trial is defined as the trial in which the firing rate of pool $\mathrm{F}$ is $>10 \mathrm{~Hz}$ and surpasses the mean firing rate of pool $\mathrm{H}$ by at least $5 \mathrm{~Hz}$. In this case, the network eventually falls into the attractor that represents percept of the face image. When the system is in the oscillatory region, a correct trial is defined as the trial in which the firing rate of pool $\mathrm{F}$ is $>10 \mathrm{~Hz}$ and surpasses first the firing rate of pool $\mathrm{H}$ by at least $5 \mathrm{~Hz}$. Decision times are defined as the times for which these criteria are first fulfilled minus the onset time of $3000 \mathrm{~ms}$ in the withoutadaptor case and minus the first $5200 \mathrm{~ms}$ in the with-adaptor case. When the system is in the bistable region, the firing rates are defined as the maximum value of the winning population's neural activity and minimum value of the losing population's neural activity, averaged over correct trials for each level of adaptation and sensory evidence (see Fig. 9A). When the system is in the oscillatory region, the firing rates are the maxima and the minima of the neural activity of the winning and losing population, respectively, in the period that the first win occurs, averaged over correct trials for each level of adaptation and sensory evidence.

Stimulation protocol D: prediction: time dominance, coefficient of variation. In this part, the time dominance and its coefficient of variation (CV) were examined after various levels of neuronal adaptation. This study presents an experimental prediction that can be tested to verify our findings. For this purpose, we calculated the mean time dominance of the percept of the hand and face images when an ambiguous composite image of both is presented for $5 \mathrm{~min}$ to subjects. We simulated this proposed experiment, having the same parameters for which the model coincides with the Cziraki et al. (2010) experiment, and defined the expected mean dominance time of perceiving the images and its coefficient of variation. The stimulation protocol is then given by $v_{\mathrm{H}}=v_{\mathrm{F}}=$ $v_{\text {ext }}$ for $0 \leq t<500 \mathrm{~ms}$ and $v_{\mathrm{H}}=v_{\mathrm{F}}=v_{\mathrm{ext}}+\lambda$ for $500 \leq t<300,000 \mathrm{~ms}$, where $\lambda=50 \mathrm{~Hz}$.

We did the same for other values of $\lambda(\lambda=30$ and $40 \mathrm{~Hz})$ for which the model coincided with the behavioral data of Cziraki et al. (2010) for same recurrent connectivity $W_{+}=1.65$. The mean dominance time is defined as the mean of periods of time starting when the firing rate of the winning neural population is $>10 \mathrm{~Hz}$ and surpasses the firing rate of the losing one by at least $5 \mathrm{~Hz}$, until the difference between them becomes $<5 \mathrm{~Hz}$. The result arises from one trial that we run for $5 \mathrm{~min}$. We repeat this protocol for different levels of adaptation, $g_{\mathrm{AHP}}$, and we concentrate our interest in its values for which the model coincides with Cziraki et al.'s (2010) experiment.

Simulations. To integrate the system of coupled differential equations that describe the dynamics of all cells and synapses, we used a second order Runge-Kutta routine (Press et al., 2007) with time step of $0.02 \mathrm{~ms}$. In each trial, the mean firing rate of each neuronal population was calculated by dividing the number of spikes emitted in a $50 \mathrm{~ms}$ window by its number of neurons and by the window size. The time window was sliding with a time step of $5 \mathrm{~ms}$. From the spiking model we also extracted the spiking times of 10 neurons from each of the four populations of the network to obtain a rastergram (see Fig. 5). Other measurements presented in this paper are calculated from the mean firing rate of the neuronal pools and are described when needed.

The spiking neural model was programmed with $\mathrm{C}++$ programming performed by Marco Loh (Department of Technology, Computational Neuroscience, Pompeu Fabra University, Barcelona, Spain), Daniel Martí (Center for Neural Science, New York University, New York, NY), and Gustavo Deco (Department of Technology, Computational Neuroscience, Pompeu Fabra University, Barcelona, Spain) and transformed adequately to the needs of the current work by the authors. For analyzing the outputs of the spiking simulations, scripts in Matlab R2007b were written and used and are available from the authors upon request.

Parameter range of $\mathrm{W}_{+}$and $\mathrm{g}_{A H P}$. To define the parameter space of recurrent connectivity weights $W_{+}$and afterhyperpolarization conductance $g_{\mathrm{AHP}}$, we investigated the behavior of the network when zero additional external input is given to its neuronal selective populations. In this case, neurons receive only background input and the firing rate of the neuronal populations should stay at low levels by increasing level of adaptation. The constraint taken into account was that the network should remain in a spontaneous state, defined as the state when mean firing rates of neuronal pools do not surpass the threshold of $10 \mathrm{~Hz}$. The resulting parameter space is shown in Figure 3.

\section{Results}

We present a biologically inspired neural model of leaky integrate-and-fire neurons with spike-frequency adapting mechanism implemented to investigate the role of neuronal adaptation in decision making. We start our theoretical/computational investigation with the examination of and comparison with the behavioral data of a high-level, adaptation-related aftereffects experiment (Cziraki et al., 2010) to understand the driving force of the perceptual decision process. Adaptation-related aftereffects refer to the impact of the previous presentation of the adaptor stimulus on the perceptual decision related to a subsequently presented stimulus. The experimental data show that prolonged exposure to complex high-level stimuli, such as faces or hands, biases the perceptual decisions of a subsequent ambiguous facehand composite stimulus away from the adaptor category, indicating that adaptation exists at a high-level of the visual processing hierarchy. Here, we hypothesize that neural frequency adaptation underlies this adaptation-related aftereffect. Even more, our simulation results support the hypothesis that neural frequency adaptation in this task is large enough so that the dynamical working regime of the network is in the oscillatory regime, indicating that the underlying model is not just a bistable model, as usual in the decision-making modeling literature. 


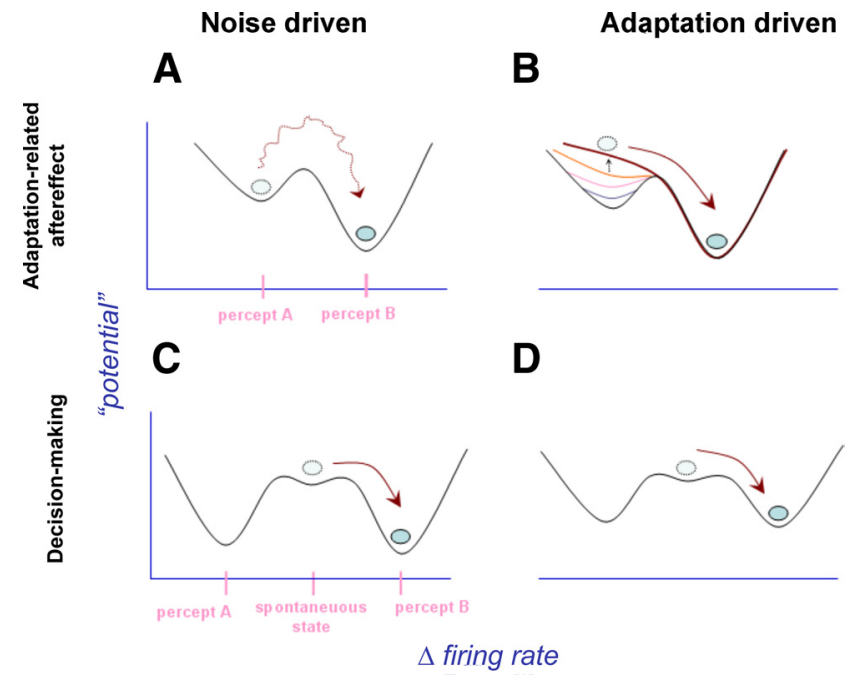

Figure 4. $\quad \boldsymbol{A}, \boldsymbol{B}$, Adaptation-related aftereffect. $\boldsymbol{A}$, Transition between the two different percepts of an ambiguous stimulus is viewed as noise-driven transition between two basins of attractors of the neural network. $\boldsymbol{B}$, Adaptation-driven, noise-sensitive fluctuations lead to oscillations. C, D, Decision-making. C, Noise-driven decision taken in a typical decision-making task of two choices. $\boldsymbol{D}$, Same procedure, but with adaptation implemented in the network, resulting in shallower basins of attractors.

We propose the hypothesis that the mechanism underlying the decision process in the adaptation-related aftereffect experiments should also be relevant and generally observable during decision making without any initial adaptor. For this purpose, we simulated a typical binary decision-making experiment without previous adaptor stimulus, using the same parameters for which the model coincides with the experimental data of Cziraki et al. (2010). We found that decision times are shorter for higher levels of neuronal adaptation, a finding that corresponds to the behavioral findings of the original study. We therefore conclude that a high level of neural adaptation in the network leads to a more efficient performance by accelerating the decision. In addition, we found that if an adaptor precedes the presentation of the composite-target stimulus, decision times are even shorter. Our results are consistent with an ecological relevance of adaptation for the prompt reactions, when a sudden change occurs in the environment to which a subject is adapted.

We considered two scenarios to understand the mechanism underlying the decision process in the adaptation-related aftereffect experiment (Cziraki et al., 2010). One is noise-driven switch due to probabilistic spiking times of neurons and the other is adaptation-driven transition arising from spike-frequency adaptation due to afterhyperpolarizing (AHP) $\mathrm{Ca}^{2+}$-activated $\mathrm{K}^{+}$currents. Both noise and adaptation mechanisms are implemented in the neuronal network. With increasing level of adaptation (AHPconductance), the network shifts from a noise-driven to an adaptation-driven modus when both neuronal selective populations are stimulated. Our initial goal is to learn which of the two mechanisms is the main driving force responsible for the perceptual switch from one percept (adaptor stimulus) to the novel percept when the ambiguous stimulus is presented at cue's end of the high-level adaptation-related aftereffects task.

Within the theoretical framework of the network that we use, each of the two competing percepts of the ambiguous stimulus can be viewed as a stable state of the neuronal dynamics (i.e., an attractor) (Fig. 4A, where percept $\mathrm{A}$ represents the percept of hand and percept $B$ represent the percept of face). Noise in our model is considered to be the source of Poisson spikes inputs amplified by the fact that the network has a limited number of neurons. Fluctuations arise in its time course and in the system's outcome on each trial. Due to the finite size effect, noise plays a crucial role in decision-making tasks by destabilizing the network's stable states, leading to the transition to other state (Fig. $4 \mathrm{~A}$, percept B). The escape from one basin of attraction to the other could be described as a Kramers' escape problem, which is applied in many problems in physics and which deals with noise-activated escape (Kramers, 1940). The mechanisms of spike-frequency adaptation is thought to be related to a slow $\mathrm{Ca}^{2+}$-activated $\mathrm{K}^{+}$current, called afterhyperpolarization current $\left(I_{\mathrm{AHP}}\right)$ because it changes after hyperpolarization of each spike. It is implemented in the network by adding an $I_{\mathrm{AHP}}$ current into the dynamical equation of the neuronal membrane potential, resulting in a gradual decrease of the firing rate of the dominant neuronal population. This effect is shown schematically in Figure $4 \mathrm{~B}$. Higher levels of neuronal adaptation result in destabilizing the initially stable states (percepts A and B), leading eventually to an oscillation between the two different network states. The adaptation mechanism may coexist with the noise but when adaptation is high, noise contributes only to the randomness of the oscillations due to the fluctuations arising from the finite-size effect, whereas when there is no adaptation, noise is the underlying mechanism.

Figure 4, $A$ and $B$, corresponds to the model with an adaptor stimulus preceding one of the target stimuli (for instance, $A$ ). In this case, there are only the two stable states in the system corresponding to each percept with shallower basin of the attractor corresponding to the category of the adaptor (i.e., A). In Figure 4, $C$ and $D$, a typical binary decision-making task is captured schematically. In this case, there is no adaptor stimulus presented prior the target. The system is initially in its spontaneous state. When both selective pools, corresponding to the two interpretations (or conflicting percepts of an ambiguous stimulus), are simultaneously stimulated, the competition between them leads eventually to one of them winning. In Figure $4 C$, the decision is taken due to noise in the network. In Figure $4 D$, the same is shown but with adaptation added to the system, resulting in shallower basins of attractors for both selective pools.

\section{Model analysis: bifurcation diagrams}

When the model replicates the presentation of an ambiguous stimulus, noise-driven fluctuations lead to switches between the two competitive interpretations of an ambiguous stimulus and adaptation-driven fluctuations lead to oscillations of the system, depending on the magnitude of the afterhyperpolarization conductance (level of adaptation). To define the underlying driving mechanism of decision making during adaptation-related aftereffects, we first examined the bifurcation diagram of the system in the noise-free model (when $N$ is large). The bifurcation diagram (Fig. 5) displays the behavior of the mean firing rate of one of the two selective populations (for instance, the $\mathrm{H}$ pool) as a function of the level of adaptation in the network. We distinguish two regimes, the bistable regime, in which transitions from one percept to the other are noise-driven, and the oscillatory regime, in which transitions are adaptation-driven. The bifurcation point is where the model passes from one regime to the other.

Therefore, after determining the parameter space for $W_{+}$and $g_{\text {AHP }}$ for which the system remains in the spontaneous state in the absence of external input (see Materials and Methods) (Fig. 3), we followed stimulation protocol A (see Materials and Methods). This parameter space also constrains the upper limit of the level of adaptation. In Figure 5, two regions are delineated by the bifurcation point $g_{\text {bif }}=3.35 \mathrm{nS}$. Before the bifurcation point, the 


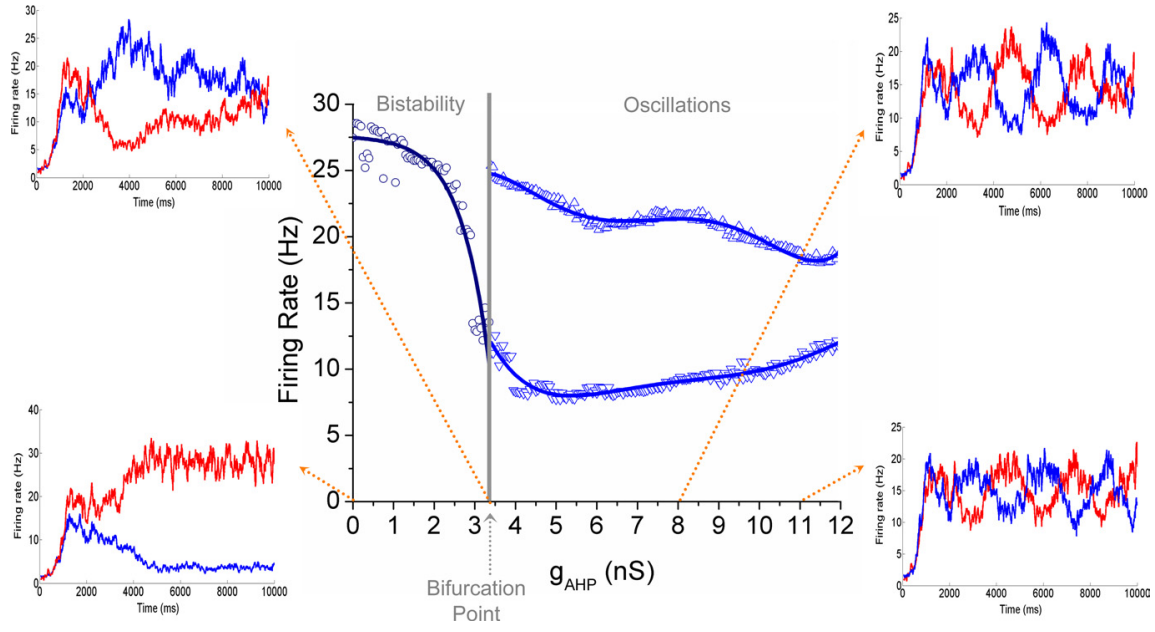

Figure 5. Bifurcation diagram for the noise-free model ( $N=4000$ neurons) as a function of level of adaptation. The system passes from bistability to an oscillatory regime at the bifurcation point $g_{\mathrm{bif}}=3.35 \mathrm{nS}$. Center graph, left, The firing rate of the dominant population at the end of the simulation is plotted for each $g_{\text {AHP }}$ (circles). Center graph, right, The mean maximum (upward-pointing triangles) and minimum (downward-pointing triangles) values of the same population's oscillatory activity are plotted. The lines are fits of the spiking simulations. Small graphs, The mean firing rates of pools $F$ (blue) and $\mathrm{H}$ (red) are plotted as a function of time for $g_{\mathrm{AHP}}=0, g_{\mathrm{bif}}, 8$, and $11 \mathrm{nS}$. finite-sized noise effects are relevant and therefore switches in the bistable regime are possible, becoming gradually more probable as approaching the bifurcation point. In the oscillatory region, noise induces randomness in the frequency of the oscillations.

In addition, we performed simulations for different levels of external stimulus $\lambda$ to show how the system transits from a spontaneous state to an oscillatory regime. Recurrent connectivity was $W_{+}=$ 1.65 and neuronal adaptation was $g_{\mathrm{AHP}}=$ 10 nS. In Figure 6, two regions are delineated by the bifurcation point $\lambda_{\text {bif }}=5 \mathrm{~Hz}$. In the spontaneous regime, the mean firing rate of one selective population is plotted (circles). In the oscillatory regime, the maximum (upwards-pointing triangles) and minimum (downwardpointing triangles) values of the first winning population's firing rate are plotted.

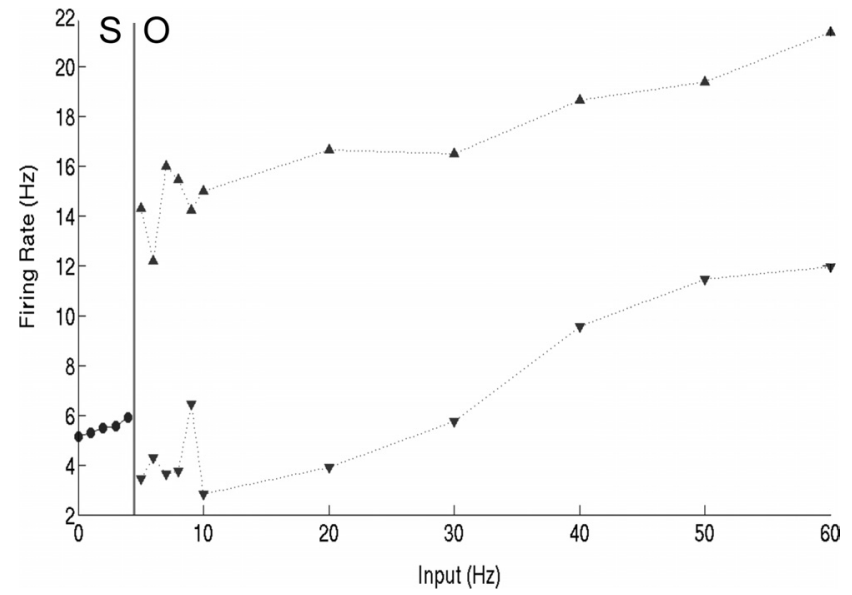

Figure 6. Bifurcation diagram for the noise-free model ( $N=4000$ neurons) as a function of external stimulus. The level of neural adaptation is $g_{\exp } \equiv g_{\text {AHP }}=10 \mathrm{nS}$. The system passes from a spontaneous state (S) to an oscillatory regime (0) when $\lambda \equiv \lambda_{\text {bif }}=5 \mathrm{~Hz}$. Left, Mean firing rate of one selective population (circles). Right, Maximum (upward-pointing triangles) and minimum (downward-pointing triangles) values of the first winning population's firing rate.

mean firing rate of the dominant perceived population at the end of the task, $t=10,000 \mathrm{~ms}$, is plotted (circles). After the bifurcation, bistability vanishes and an oscillatory regime appears. The maximum (upward-pointing triangles) and minimum (downward-pointing triangles) values of the mean firing rate of pool $\mathrm{H}$ are plotted. The maxima and minima are obtained after smoothing the firing rate of the population in a $500 \mathrm{~ms}$ period, using $50 \mathrm{~ms}$ sliding windows. In the noise-free model, or, to be more precise, in the model of low noise such that does not change significantly the result, and in the bistable regime, the system cannot escape from the stable state and therefore cannot switch from one state to the other. In contrast, in the oscillatory regime, there are no stable states at all. Transitions from one state to the other arise in a periodic rhythm depending on the level of adaptation. When the number of active neurons is small,
Simulations in comparison with psychophysical data

Our next step was to find the level of adaptation for which the modeling results from the spiking-neuron simulations coincide with the behavioral data (Fig. $1 B$ ). To replicate Cziraki et al.'s (2010) experiment, we used an attractor network of leaky integrate-and-firing neurons with spike-frequency adaptation implemented using stimulation protocol B (see Materials and Methods). In our model, two selective pools are thought to represent populations of neurons in high-level cortical areas FFA and EBA. Having the bifurcation diagram (Fig. 5) as a reference, we calculated the mean ratio of face and hand responses after hand adaptation in EBA for different values of level of adaptation $g_{\mathrm{AHP}}$. Switch in this case is the percept of the novel image when both stimuli are presented to the subject via their composite ambiguous image after adaptation. The result is demonstrated in Figure $7 A$. More trials were judged as faces after hand adaptation, with mean percentage being $73 \%$. The level of adaptation is $g_{\exp } \equiv g_{\text {AHP }}=10 \mathrm{nS}$. Since $g_{\exp }>g_{\text {bif }}$, we conclude that the working point of the network is in the oscillatory regime. The mean ratio of face responses after hand adaptation is equivalent to hand responses after face adaptation since our network is symmetric. The rastergram (Fig. $7 B$ ) shows the spiking times of 10 neurons from each of the four neural populations during one trial when the results of the model coincided with that of the behavioral data, $g_{\text {exp }}$. Interestingly, this level of adaptation is close to the value $g_{\mathrm{AHP}}=7.5 \mathrm{nS}$ used in Liu and Wang (2001) and Deco and Rolls (2005), where the same parameters of $\left[\mathrm{Ca}^{2+}\right]$ dynamics were used to study the dynamics of one IF neuron (Liu and Wang, 2001) and a network of spiking neurons (Deco and Rolls, 2005b). In Figure $7 C$, the time course of the mean firing rates is plotted for all four neuronal populations on one trial in which pool $\mathrm{F}$ wins the competition at $5200 \leq t \leq 5400 \mathrm{~ms}$. The system initially reaches a stable state and then, when both pools are stimulated, competition will eventually lead to oscillations due to high level of neuronal adaptation. We thus predict that if the targetambiguous composite stimulus is persistently presented, in time subjects will experience rivalry, revealed as an oscillation in perception from one type to the other. 


\section{Parameter search supporting the results}

The results demonstrated in Figure 7 arise from a specific set of the three main free parameters: $W_{+}=1.65, \lambda_{\text {adaptor }}=200$ $\mathrm{Hz}$, and $\lambda=50 \mathrm{~Hz}$. This set is only one example from a variety of parameter sets for which the results of the spiking simulations coincide with the behavioral data. It was found after performing an analytical parameter search while keeping the total number of neurons in the network constant and equal to $N=2000$ neurons. The range of values of recurrent connectivity weight $W_{+}$was $1.6 \leq W_{+} \leq 1.8$, defined by both the region for which there can be decision states when stimulus is applied to selective pools (Martí et al., 2008) and the region where the network remains at the spontaneous state when no additional external stimulus is applied to the selective pools determining, in parallel, the upper limit of level of adaptation, $g_{\mathrm{AHP}}$ (Materials and Methods) (Fig. 3). The range of external input to the two selective pools $\mathrm{H}$ and $\mathrm{F}$ in the second part of stimulation protocol B was $10 \leq \lambda \leq 60 \mathrm{~Hz}$, defined by the constraint that decision states and/or oscillations exist depending on adaptation, $g_{\mathrm{AHP}}$. As for $\lambda_{\text {adaptor }}$, the range of values was $\lambda \leq \lambda_{\text {adaptor }} \leq 60 \mathrm{~Hz}$. The adaptor was a high-contrast hand or face and the test stimulus was a composite image of a hand and a face different from the adaptors and of lower contrast. In our simulations, we started from the point where both the adaptor and the test stimuli are equally excited by $\lambda$ and $\lambda_{\text {adaptor }}$, respectively. We continued by studying the response of the system by manipulating the $\lambda_{\text {adaptor }}$. We chose a frequency of $60 \mathrm{~Hz}$ as an upper limit, but afterward examined the effect of an increase in the adaptor's stimulus amplitude.

From the parameter search, with the above-defined constraints, we found that the model coincides with the behavioral results for $1.6 \leq W_{+} \leq 1.65$ and, whenever this happened for different test stimuli $\lambda$ and adaptor stimuli $\lambda_{\text {adaptor }}$, the underlying mechanism was adaptation-driven. By varying input $\lambda$, the bifurcation point changes for a given value of the $W_{+}$. From the cases for which the model coincides with the experiment, we found that by increasing $\lambda$ while keeping $W_{+}$and $\lambda_{\text {adaptor }}$ constant, the $g_{\exp }$ decreases but so does also $g_{\text {bif }}$, although the difference $g_{\exp }-g_{\text {bif }}$ remains constant with $g_{\exp }$ being always in the oscillatory region ( $g_{\exp } \gg g_{\text {bif }}$ ). By increasing $W_{+}$while keeping $\lambda$ and $\lambda_{\text {adaptor }}$ constant, the $g_{\text {bif }}$ increases but so does $g_{\text {exp }}$. Again, the difference $g_{\exp }-g_{\text {bif }}$ remains constant with $g_{\exp }$ being always in the oscillatory region. We also investigated the effect of increasing noise by decreasing the number of total neurons in the network, resulting in stronger, finite-sized noise. For fewer neurons in the network, $g_{\text {bif }}$ is constant and $g_{\exp }$ is smaller; thus, the difference $g_{\text {exp }}-g_{\text {bif }}$ decreases, meaning that the role of noise increases. However, noise never becomes the main driving force. The working point of the system remains $g_{\exp }$ in the oscillatory regime.

Finally, we studied the effect of the adaptor's stimulus strength $\left(\lambda_{\text {adaptor }}\right)$. We saw that by increasing $\lambda_{\text {adaptor }}$ while keeping con- stant $W_{+}, \lambda$, and $N$, the difference $g_{\exp }-g_{\text {bif }}$ gradually decreased. Since the upper limit of $\lambda_{\text {adaptor }}$ was selectively chosen without any specific constraint, this decrease would probably lead to $g_{\exp }$ $\leq g_{\text {bif }}$, which means that the decision making is driven by noise. Hence, Figure $8 \mathrm{~A}$ shows the decrease of $g_{\exp }$ as a function of adaptor stimulus, $\lambda_{\text {adaptor }}$, with all other parameters equal to the ones used for Figure 7. We can clearly see that the $g_{\exp }$ never reaches the bifurcation point $g_{\text {bif }}=3.35 \mathrm{nS}$ even for extreme values of $\lambda_{\text {adaptor }}$. In Figure $8 B$, the same point is demonstrated. The percentage performance of face responses after hand adaptation is plotted for different levels of adaptation as a function of the initial external input to pool $\mathrm{H}, \lambda_{\text {adaptor. From the previous }}$ discussion and this last study, we can be sure that in this highlevel, adaptation-related aftereffects experiment, the underlying mechanism of the decision-making process when the composite ambiguous image is presented at the end of the cue is adaptationdriven and the working point of the system is in the oscillatory regime.

\section{Decision making and decision certainty}

Decision making in binary choices has been studied extensively, both experimentally and theoretically, to understand its underlying mechanisms. Lately, there has been an increased interest in the confidence in decisions and it has been seen that reaction times of the decisions are shorter on easy than on difficult trials (Kim and Shadlen, 1999). In addition, it has been shown that when the input to the selective-neuron pool of the network in the 
A

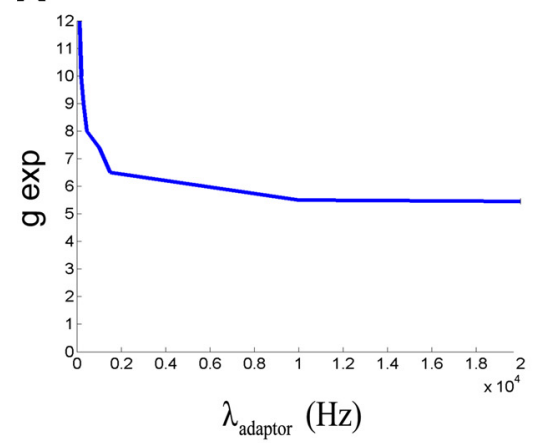

B

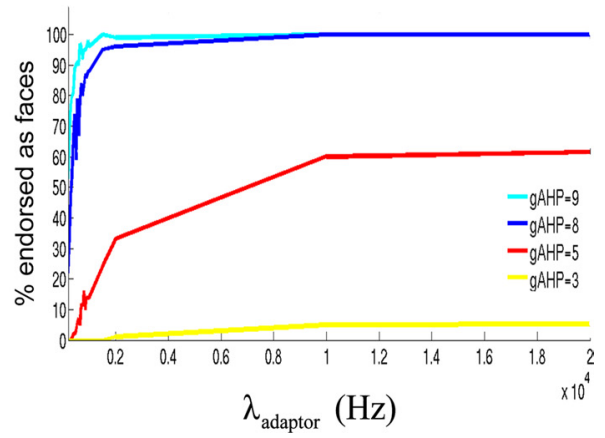

Figure 8. $\quad A$, Value of the adaptation parameter for which the results of the model coincide with the experimental behavioral data, $g_{\text {exp }}$ as a function of the initial external input, $\lambda_{\text {adaptor }}$ to the neuronal pool $H$. The total number of neurons in the network is 2000 and recurrent connectivity is $W_{+}=1.65$. The stimulus to both pools, F and H, during the last $200 \mathrm{~ms}$ of the cue, representing the presentation of the ambiguous stimulus, is $\lambda=50 \mathrm{~Hz}$. The $g_{\exp }$ never reaches the bifurcation point $g_{\text {bif }}=3.35$ $\mathrm{nS}$ even for extreme values of $\lambda_{\text {adaptor }} \boldsymbol{B}$, The ratio of face responses after hand adaptation is plotted for different levels of adaptation, $g_{\text {AHp }}$ as a function of the initial input, $\lambda_{\text {adaptor }}$.

adaptation-free model is increased, decision times are also shorter for high-input versus for low-input situations (Martí et al., 2008). Nevertheless, the effect of the firing-rate adaptation mechanism in decision-making processes has not yet been studied. Next, we studied this effect by using the same spiking network with the same parameters as we used to define the underlying mechanism in the high-level adaptation-related aftereffects task of Cziraki et al. (2010).

We followed stimulation protocol C (see Materials and Methods) for different levels of adaptation. We began our study without having any adaptor in the task, meaning that the experimental task was to present an ambiguous face-hand composite stimulus (Cziraki et al., 2010) that changes over trials to either the face or the hand image, therefore increasing sensory evidence available about one of the interpretations of the stimulus and decreasing its ambiguity. Psychophysically, the measure would be the ratio of trials endorsed as faces. Neurophysiologically, it would be the neural activity of the cortical areas EBA and FFA and the decision times (i.e., the times needed for perceiving the stimulus as hand or face) as a function of the level of sensory evidence (i.e., the increase of the amount of the sensory evidence of one interpretation of the composite image). We simulated this task by externally increasing the input to neurons of selective population $\mathrm{F}$ by $\Delta \lambda$ while simultaneously decreasing the external input to neurons of selective populations $\mathrm{H}$ by $\Delta \lambda$ over trials. We calculated the mean firing rate of both pools as a function of the total external input to the network, $\Delta \mathrm{I}$, averaged on correct trials from 1000 simulations. The total external input $\Delta \mathrm{I}$ is the difference in spikes per second summed across all synapses to each neuron between the external input $\lambda+\Delta \lambda$ to pool $\mathrm{F}$ and $\lambda-\Delta \lambda$ to pool $\mathrm{H}$. Correct trials are defined as the trials when the mean firing rate of pool $\mathrm{F}$ surpasses first and adequately the mean firing rate of pool H (see Stimulation protocol C). Then we ran 1000 trials for different levels of adaptation, $0 \leq g_{\text {AHP }} \leq 12$.

Figure $9 A$ shows the neural activity (mean firing rate $\pm 1 / 2 \times$ $\mathrm{SD}$ ) of pools $\mathrm{F}$ (solid lines) and $\mathrm{H}$ (dashed lines) on correct trials. In Figure $9 B$, the percentage performance $\pm 1 / 2 \times$ estimated error over 1000 trials (estimated error $=\sqrt{\text { performance } / 1000}$ ) for different levels of adaptation is plotted. Figure $9 C$ shows the mean decision times for different levels of adaptation $\pm 1 / 2 \times \mathrm{SD}$ on correct trials and the activities of the neuronal population in one single trial in the most difficult decision case $(\Delta \mathrm{I}=0)$ and in the

most easy one $(\Delta \mathrm{I}=60)$ without adaptation $\left(g_{\mathrm{AHP}}=0 \mathrm{nS}\right)$ and for the level of adaptation for which the model coincides with Cziraki et al.'s (2010) experiment $\left(g_{\text {AHP }} \equiv g_{\text {exp }}=10 \mathrm{nS}\right)$.

The first important conclusion from Figure 9 is that all levels of adaptation show the same behavior as a function of the level of sensory evidence: Neural activity of the F pool increases monotonically as a function of level of sensory evidence and its variability decreases. The variability in the firing rates is higher in difficult trials, reflecting that the system is noisier since there is less available sensory evidence. The difference between the neural activity of the winning and the losing neural population increases with the level of sensory evidence, reflecting the increase in certainty as the task gets easier. In difficult trials, low certainty in the decision is also reflected in the performance. The percentage of correct choices, which starts from chance level (50\%) showing the ambiguity of the stimulus when there is equal level of sensory evidence applied to the selective neuronal populations, increases as the task gets easier (Fig. 9B). The performance increases monotonically as a function of the level of sensory evidence.

Second and more interestingly, we observed that, for higher levels of adaptation, the decision times are shorter for all the range of $\Delta \mathrm{I}$ (Fig. 9C). Furthermore, the variability in decision times caused by the random spiking of the neurons in the network is high in difficult decision cases and decreases as certainty increases. The activity of the winning neuronal population is decreasing as adaptation increases and the activity of the losing population increases, indicating that the certainty decreases but decision times are smaller. In addition, the rate of increase of the performance curve depends on the level of adaptation. In conclusion, these results show that adaptation in the context of decision making could serve to the speed-accuracy trade-off.

Our findings show that neuronal adaptation in the firing rate of the competitive neuronal population lead to faster discrimination among them. After calculating the balance of the excitationinhibition of the selective populations, we found that higher levels of neural adaptation lead the system to become more imbalanced, resulting in shorter decision times. This result can also be explained schematically if we consider the representation of the two percepts seen as basins of attractors in a bistable system (Fig. 4). In the network, noise arising from the probabilistic spiking times exists with and without neural adaptation. If there is no adaptation in the system, then a certain time is needed for the system to move from one state to the other. When adaptation is added into the system having the same level of noise, there is a higher probability that the system will move from one state to the other because the basin of attraction of the dominant perceptual state is becoming shallower in parallel and the other interpretation can win the competition earlier. Higher levels of neural adaptation lead to even shallower basins of the dominant attractor and the transition is even faster. When one neuron receives a Poisson spike train input, its firing rate decreases with increasing adaptation, which also increases the variability of its output spike trains (Liu and Wang, 2001). In a neural network, this output spike train with higher variability when adaptation is implemented is an 
additional noise into the postsynaptic neurons, resulting in an increase in the noise and eventually to a faster transition.

Next, we applied the same adaptor stimuli to the current system as in Cziraki et al.'s (2010) experiment (Stimulation protocol C; with adaptor). We were interested in the effect of adaptation on the decision times. Figure 10 shows decision times for the adapted (dashed lines) and unadapted (solid lines) conditions for various levels of adaptations $\left(g_{\exp }-1 \leq\right.$ $\left.g_{\text {AHP }} \leq g_{\exp }+1\right)$. For the adapted case, the behavior is the same as in the unadapted case. Decision times decrease analogous to the level of sensory evidence for all levels of adaptation, with a smaller slope than in the unadapted case. In addition, decision times decrease as magnitude of adaptation increases. Interestingly, although Figure 10 shows that when a subject is adapted to a perceptual interpretation of an ambiguous composite stimulus, the percept of the other interpretation occurs faster $(\Delta \mathrm{I}=0)$ than when there is no adaptor. From an ecological point of view, it is extremely important to be able to react as fast as possible to a sudden change in the environment. As we increase the magnitude of adaptation over trials, a novel percept (in this case, the opposite interpretation of the target stimulus) is more likely to occur. Our results suggest that it also happens faster. It is also logical from a theoretical point of view since the activity of the adapted neuronal population is suppressed and it is easier for the competitive neuronal population to win the competition, leading to faster reaction times.

\section{Discussion}

In the current work, we investigated the role of the spike-frequency adaptation in the context of decision making. We used a biophysically realistic competition model of decision making with an implemented spike-frequency adaptation mechanism. Decision making and its underlying mechanisms is a common field of interest among neuroscientists during the last few decades but the role of neural adaptation in this context is still unknown. Our work addresses some fundamental aspects of adaptation effects in decision making.

We began our study by considering behavioral data from a high-level adaptation-related aftereffects experiment (Cziraki et al., 2010). In this experiment, it was shown that prolonged adaptation to a complex stimulus (e.g., an image of a hand) leads to category-specific aftereffects during the perception of ambiguous stimulus composites (a composite image of a hand and a face). From a theoretical point of view, we studied the dynamics of the perceptual switch from the adaptor category to the novel, opposite category by comparing two scenarios: noise- and adaptation-driven transition. When an ambiguous stimulus is presented, noise-driven fluctuations lead to switches from one
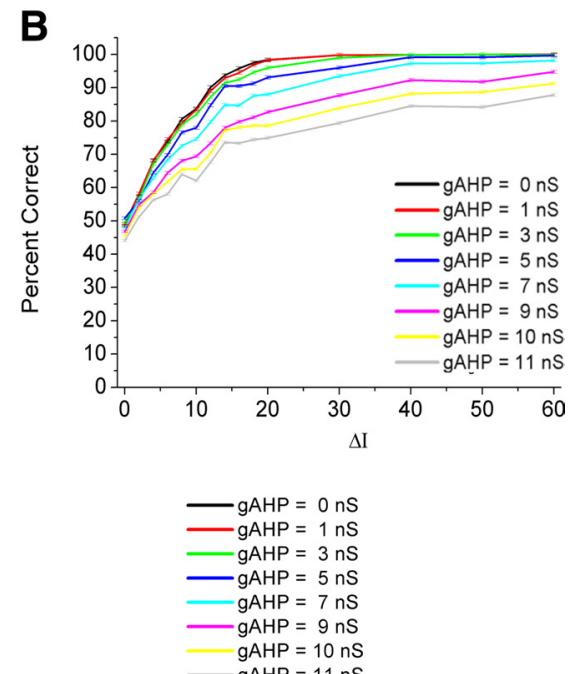

GAHP $=11 n S$

Figure 9. Decision making without an adaptor preceding the decision-making task for different levels of adaptation. $\boldsymbol{A}$, Mean firing rates of the winning pool (solid lines) and of the loosing pool (dashed lines) averaged on correct trials over 1000 simulations. $\boldsymbol{B}$, Percentage of correct trials as a function of the level of sensory evidence. $\boldsymbol{C}$, Decision times when the selective neuronal pool receiving increased level of sensory evidence wins competition, averaged over correct trials. Bottom left, Mean firing rate of al pools in the adaptation-free and in high-level adaptation case, both in a single trial of the most difficult case for decision making $(\Delta \mathrm{I}=0 \mathrm{~Hz})$. Bottom right, The same plots from one single trial for the easiest case $(\Delta \mathrm{I}=60 \mathrm{~Hz})$.

percept to the other. These fluctuations arise in the network due to the probabilistic spike time of the neurons amplified by finitesized effects. Adaptation-driven transitions, on the other hand, lead to oscillations between the two different percepts, with random frequency while noise-driven fluctuations still exists in the network. We investigated the dynamical regime for which the competition model is consistent with the behavioral data. The behavioral result and our detailed parameter study, in the accepted parameter space, constrain the adaptation-driven regime to be the working point of the network.

Since we find that spike-frequency adaptation is the underlying mechanism in high-level, decision-making tasks like the high-level, adaptation-related aftereffects, we extended our study to typical binary decision-making tasks. We simulated an experiment of decision making where an ambiguous figure, like the 


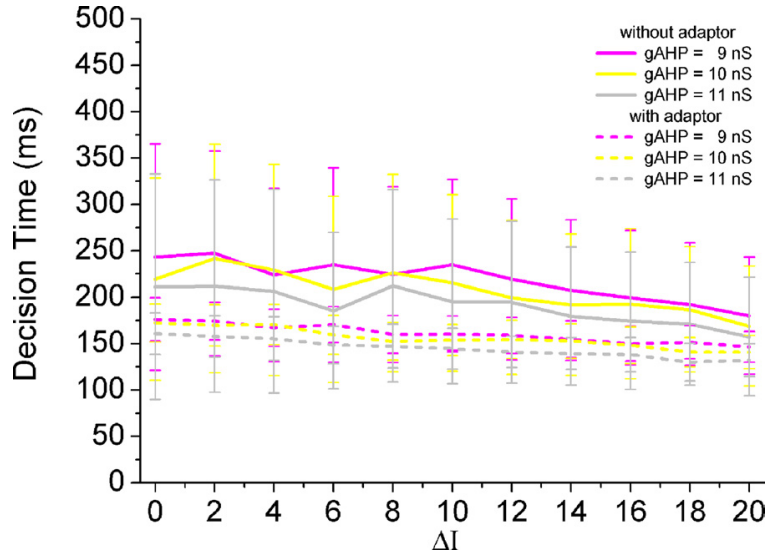

Figure 10. Decision making with (dashed lines) and without (solid lines) an adaptor preceding the decision-making task for different values of neural adaptation around the $g_{\exp }$ value. With adaptor, the behavior is the same as without adaptor, but decision times are shorter.

one presented in Cziraki et al. (2010), is presented to subjects. In this experiment, the contrast of one of the two competing percepts is increased over different sets of trials. Simulating this experiment for different levels of adaptation, we found that when neuronal adaptation is high, decision becomes faster while the rate of increase of the performance curve decreases, indicating the role of neuronal adaptation in the speed-accuracy trade-off. With increasing levels of neural adaptation, the balance between excitatory and inhibitory currents received at the synapses of the selective populations decreases, resulting in shorter decision times. In addition, when an adaptor precedes the decisionmaking task, decision is even faster for the same level of adaptation. The output of the spiking model we use could be tested in psychophysical and neurophysiological experiments. Our results are also consistent with an ecological point of view, since it is important to be able to react quickly when new environmental information is presented.

Finally, we integrate our work by presenting testable predictions via experimental tasks that may verify our findings. For the decision-making task presented above, an increase in the reaction times should be observed if the $\mathrm{Ca}^{2+}$-activated $\mathrm{K}^{+}$channels can be gradually blocked. We also propose another task. The composite ambiguous figure of a hand and a face (Cziraki et al., 2010) is presented to subjects continuously for $5 \mathrm{~min}$ and the psychophysically mean time dominance and coefficient of variation are measured. We mimic this task by having the same parameters as those in Cziraki et al. (2010). We calculate the mean dominance time of perceiving the hand or the face and its coefficient of variation (Stimulation protocol D). The result is shown in Figure 11. In Figure 11, the green line corresponds to $\lambda=50 \mathrm{~Hz}$, which is the external stimulus mainly used in this paper, but calculations were also performed for $\lambda=30 \mathrm{~Hz}$ and $\lambda=40 \mathrm{~Hz}$ (with same recurrent connectivity), for which the model coincided with the behavioral data of Cziraki et al.'s (2010) experiment. Hence, psychophysically, we expect to measure a mean dominance time, $T$, approximately in the range of $2 \leq \bar{T} \leq 6 \mathrm{~s}$ and its coefficient of variation in the range of $0.5<\mathrm{CV} \leq 1$. We therefore conclude that the ambiguous composite stimulus is indeed a rivalrous stimulus. Preliminary experimental data further confirm our prediction. The same ambiguous composite stimulus was presented for $30 \mathrm{~s}$ and subjects $(n=3)$ were asked to report which image they perceived by pressing a button. Subjects experienced alternations in their perception with a mean time dominance of

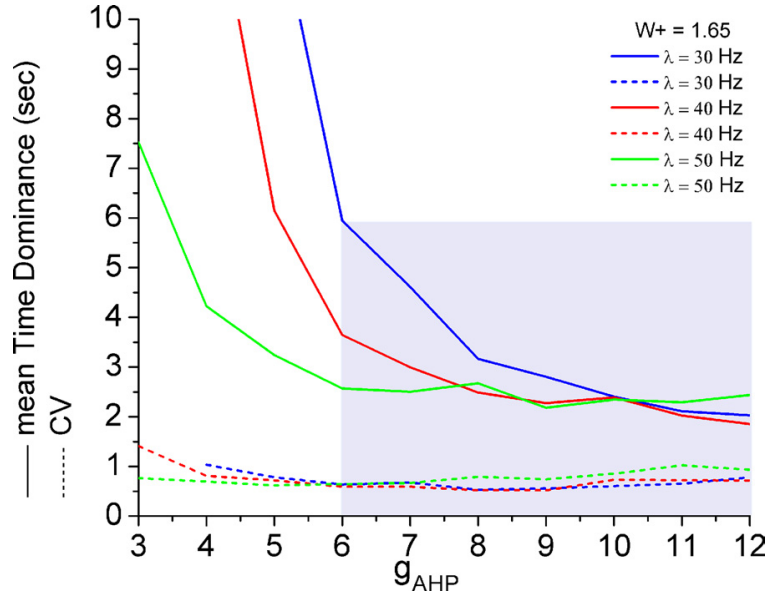

Figure 11. Mean dominance time and coefficient of variation for different levels of adaptation when the ambiguous composite figure of the hand and the face is presented, having the same parameters as the ones coinciding with the behavioral data from Cziraki et al.'s (2010) experiment.

$T_{\exp }=4.5 \mathrm{sec}$ and coefficient of variation $\mathrm{CV}=0.52$. Naturally, more subjects are needed to be quantitatively more precise but qualitatively we can conclude that the composite stimulus of our study is indeed rivalrous, since the experimental time dominance and coefficient of variation is within the typical range of rivalrous figures (Levelt, 1968). This result is also in line with previous experiments where adaptation led to unambiguous percepts of such ambiguous stimuli as the Necker cube (Long and Moran, 2007). In this study, previous exposure to an unambiguous version of the Necker cube for several minutes produced an immediate bias to perceive the ambiguous figure in the opposite configuration and led to decreased number of reversals. We note that the experimental data are also within the predicted range by our study. We therefore conclude that our hypotheses that neuronal adaptation should be relevant in Cziraki et al.'s (2010) experiment, which led to the conclusion that our model can account for modeling perceptual bistability.

Perceptual bistability emerges when an ambiguous stimulus with two mutually exclusive interpretations is perceived as the frequent alternation of these states during long-term stimulus presentation. This visual phenomenon arises in many domains of perception, such as ambiguous figures as the well known facevase stimulus, the Necker cube, or monocular rivalry, but the most extensively studied domain is binocular rivalry (for review, see Blake and Logothetis, 2002). Neurodynamical models proposed for binocular rivalry are based on a competition between two populations of excitatory neurons whose activities encode the two conflicting percepts. This competition is mediated through inhibitory neurons that lead the dominant neuronal population to suppress the activity of the competitive one, such that at aiven time only one has high activity corresponding to one of the interpretations.

Moreover, theoretical studies show that random alternations in perception in such tasks can be achieved by two mechanisms: adaptation-driven and noise-driven transitions. Neuronal adaptation implemented by spike-frequency adaptation and/or synaptic depression results in reducing the activity of the dominant neuronal population or in weakening the inhibition between the two populations of neurons, leading the nonadapted population to win the competition. In this case, even in the absence of noise, adaptation generates alternations between the competing per- 
cepts with perfect periodicity, the reason these models are called oscillator models (Lehky, 1988; Lago-Fernández and Deco, 2002; Laing and Chow, 2002; Moreno-Bote et al., 2007; Shpiro et al., 2007). In contrast, noise-driven fluctuations in the firing rates of the neuronal populations in a bistable attractor network lead to random switches between two stable attractors representing the two dissimilar percepts (Salinas, 2003; Freeman, 2005; Kim et al., 2006; Moreno-Bote et al., 2007). In these models, called noisedriven attractor models, a switch in perception cannot exist without noise. Both mechanisms have recently been studied jointly in a common neuronal competition-rate model (Shpiro et al., 2009). Shpiro et al. (2009) show that the working point of the models, considering both noise-driven and adaptation-driven mechanisms, should be at the edge of the bifurcation.

In the present paper, we considered both mechanisms within the same theoretical framework, using a spiking neuronal model to study the underlying mechanism in a high-level, adaptationrelated aftereffects task (Cziraki et al., 2010). We found that neuronal adaptation is high enough that the working point of the system is in the oscillatory regime. We therefore predict that if the target stimulus is presented continuously, subject will experience rivalry with the predicted time dominance and coefficient of variation. Preliminary experimental results confirm our prediction. Although these preliminary data are enough to qualitatively confirm our hypothesis, we understand that more subjects should be tested to provide more precise quantitatively results. The predicted dominance time calculated is inside the range of typical dominance times measured experimentally when rivalry figures are presented to subjects, but the predicted coefficient of variation can be larger than the upper bound of the typical range (Levelt, 1968).

In conclusion, our study links, for the first time, neural processes and high-level, adaptation-related aftereffects. Using a spiking neural network, including neuronal adaptation mechanism by the slow $\mathrm{Ca}^{2+}$-activated $\mathrm{K}^{+}$current, we found that neuronal adaptation is the main driving force in such high-level visual phenomena in humans. Furthermore, we have investigated the effect of this mechanism in typical binary decision-making task and found that neuronal adaptation leads to faster decisions, contributing to a speed-accuracy trade-off.

\section{References}

Abeles A (1991) Corticonics. New York: Cambridge UP.

Amit DJ (1995) The Hebbian paradigm reintegrated: local reverberations as internal representations. Behav Brain Sci 18:617-626.

Blake R, Logothetis NK (2002) Visual competition. Nat Rev Neurosci 3:13-21.

Brunel N, Wang XJ (2001) Effects of neuromodulation in a cortical network model of object working memory dominated by recurrent inhibition. J Comput Neurosci 11:63-85.

Cziraki C, Greenlee MW, Kovács G (2010) Neural correlates of high-level adaptation-related aftereffects. J Neurophysiol 103:1410-1417.

Deco G, Rolls ET (2005) Neurodynamics of biased competition and coop- eration for attention: a model with spiking neurons. J Neurophysiol 94:295-313.

Deco G, Rolls ET (2006) Decision-making and Weber's law: a neurophysiological model. Eur J Neurosci 24:901-916.

Deco G, Rolls ET, Romo R (2009) Stochastic dynamics as a principle of brain function. Prog Neurobiol 88:1-16.

Downing PE, Jiang Y, Shuman M, Kanwisher N (2001) A cortical area selective for visual processing of the human body. Science 293:2470-2473.

Freeman AW (2005) Multistage model for binocular rivalry. J Neurophysiol 94:4412-4420.

Gold JI, Shadlen MN (2007) The neural basis of decision making. Annu Rev Neurosci 30:535-574.

Kanwisher N, McDermott J, Chun MM (1997) The fusiform face area: a module in human extrastriate cortex specialized for face perception. J Neurosci 17:4302-4311.

Kim JN, Shadlen MN (1999) Neural correlates of a decision in the dorsolateral prefrontal cortex of the macaque. Nat Neurosci 2:176-185.

Kim YJ, Grabowecky M, Suzuki S (2006) Stochastic resonance in binocular rivalry. Vision Res 46:392-406.

Kramers HA (1940) Brownian motion in a field of force and the diffusion model of chemical reactions. Physica 7:284-304.

Lago-Fernández L, Deco G (2002) A model of binocular rivalry based on competition in IT. Neurocomputing 44:503-507.

Laing CR, Chow CC (2002) A spiking neural model of binocular rivalry. J Comput Neurosci 12:39-53.

Lehky SR (1988) An astable multivibrator model of binocular rivalry. Perception 17:215-228.

Levelt W (1968) On binocular rivalry. Paris: Mouton.

Liu YH, Wang XJ (2001) Spike-frequency adaptation of a generalized leaky integrate-and-fire model neuron. J Comput Neurosci 10:25-45.

Long GM, Moran CJ (2007) How to keep a reversible figure from reversing: teasing out top-down and bottom-up processes. Perception 36:431-445.

Madison DV, Nicoll RA (1984) Control of the repetitive discharge of rat CA1 pyramidal neurons in vitro. J Physiol Lond 345:319-331.

Martí D, Deco G, Mattia M, Gigante G, Del Guidice P (2008) A fluctuationdriven mechanism for slow decision processes in reverberant networks. PLoS One 3:e2534.

Moreno-Bote R, Rinzel J, Rubin N (2007) Noise-induced alternations in an attractor network model of perceptual bistability. J Neurophysiol 98: $1125-1139$.

Nordlie E, Gewaltig MO, Plesser HE (2009) Towards reproducible descriptions of neuronal network models. PLoS Comput Biol 5:e1000456.

Press WH, Teukolsky SA, Vetterling WT, Flannery BP (2007) Numerical recipes: the art of scientific computing, Ed 3. New York: Cambridge UP.

Salinas E (2003) Background synaptic activity as a switch between dynamical states in a network. Neural Comput 15:1439-1475.

Shadlen MN, Newsome WT (1996) Motion perception: seeing and deciding. Proc Natl Acad Sci U S A 93:628-633.

Shpiro A, Curtu R, Rinzel J, Rubin N (2007) Dynamical characteristics common to neuronal competition models. J Neurophysiol 97:462-473.

Shpiro A, Moreno-Bote R, Rubin N, Rinzel J (2009) Balance between noise and adaptation in competition models of perceptual bistabilty. J Comput Neurosci 27:37-54.

Smith PL, Ratcliff R (2004) Psychology and neurobiology of simple decisions. Trends Neurosci 27:161-168.

Wang XJ (2002) Probabilistic decision making by slow reverberation in cortical circuits. Neuron 36:955-968.

Wang XJ (2008) Decision making in recurrent neuronal circuits. Neuron 60:215-234.

Wong KF, Wang XJ (2006) A recurrent network mechanism of time integration in perceptual decisions. J Neurosci 26:1314-1328. 